\title{
A Determination of the Spin of the Black Hole Primary in LMC X-1
}

\section{Citation}

Gou, Lijun, Jeffrey E. McClintock, Jifeng Liu, Ramesh Narayan, James F. Steiner, Ronald A. Remillard, Jerome A. Orosz, Shane W. Davis, Ken Ebisawa, and Eric M. Schlegel. 2009. "A Determination of the Spin of the Black Hole Primary in LMC X-1." The Astrophysical Journal 701 (2) (July 29): 1076-1090. doi:10.1088/0004-637x/701/2/1076.

\section{Published Version}

doi:10.1088/0004-637X/701/2/1076

\section{Permanent link}

http://nrs.harvard.edu/urn-3:HUL.InstRepos:26526403

\section{Terms of Use}

This article was downloaded from Harvard University's DASH repository, and is made available under the terms and conditions applicable to Other Posted Material, as set forth at http:// nrs.harvard.edu/urn-3:HUL.InstRepos:dash.current.terms-of-use\#LAA

\section{Share Your Story}

The Harvard community has made this article openly available.

Please share how this access benefits you. Submit a story.

\section{Accessibility}




\title{
A DETERMINATION OF THE SPIN OF THE BLACK HOLE PRIMARY IN LMC X-1
}

\author{
Lijun Gou $^{1}$, Jeffrey E. McClintock ${ }^{1}$, Jifeng Liu ${ }^{1}$, Ramesh Narayan ${ }^{1}$, James F. Steiner $^{1}$, Ronald A. Remillard ${ }^{2}$, Jerome \\ A. Orosz ${ }^{3}$, Shane W. Davis $^{4}, \mathrm{Ken}_{\text {Ebisawa }}{ }^{5}$, and Eric M. Schlegel ${ }^{6}$ \\ ${ }^{1}$ Harvard-Smithsonian Center for Astrophysics, 60 Garden street, Cambridge, MA 02138, USA \\ ${ }^{2}$ Kavli Institute for Astrophysics and Space Research, Massachusetts Institute of Technology, Cambridge, MA 02139, USA \\ ${ }^{3}$ Department of Astronomy, San Diego State University, 5500 Companile Drive, San Diego, CA 92182, USA \\ ${ }^{4}$ Institute For Advanced Study, Einstein Drive, Princeton, NJ 08540, USA \\ ${ }^{5}$ Institute of Space and Astronautical Science/JAXA, 3-1-1 Yoshinodai, Sagamihara, Kanagawa 229-8510, Japan \\ ${ }^{6}$ Department of Physics and Astronomy, University of Texas at San Antonio, 1 UTSA Circle, San Antonio, TX 78249, USA \\ Received 2009 January 7; accepted 2009 June 19; published 2009 July 29
}

\begin{abstract}
The first extragalactic X-ray binary, LMC X-1, was discovered in 1969. In the 1980s, its compact primary was established as the fourth dynamical black hole candidate. Recently, we published accurate values for the mass of the black hole and the orbital inclination angle of the binary system. Building on these results, we have analyzed $53 \mathrm{X}$-ray spectra obtained by $R X T E$ and, using a selected sample of 18 of these spectra, we have determined the dimensionless spin parameter of the black hole to be $a_{*}=0.92_{-0.07}^{+0.05}$. This result takes into account all sources of observational and model-parameter uncertainties. The standard deviation around the mean value of $a_{*}$ for these 18 X-ray spectra, which were obtained over a span of several years, is only $\Delta a_{*}=0.02$. When we consider our complete sample of $53 R X T E$ spectra, we find a somewhat higher value of the spin parameter and a larger standard deviation. Finally, we show that our results based on $R X T E$ data are confirmed by our analyses of selected X-ray spectra obtained by the XMM-Newton, BeppoSAX, and Ginga missions.
\end{abstract}

Key words: binaries: general - black hole physics - galaxies: individual (LMC) - X-rays: binaries

\section{INTRODUCTION}

LMC X-1 was the first extragalactic X-ray binary to be discovered (Mark et al. 1969; Price et al. 1971). Its X-ray flux is quite constant, varying in intensity by $\lesssim 25 \%$ during 12 years of monitoring by the All-Sky Monitor (ASM) on board the Rossi $X$-ray Timing Explorer (RXTE; Orosz et al. 2009). The source has been observed by essentially all X-ray astronomy missions from Uhuru to Chandra (e.g., Leong et al. 1971; Cui et al. 2002). Its bolometric, isotropic luminosity is $L_{\text {bol }} \approx 2.2 \times 10^{38} \mathrm{erg} \mathrm{s}^{-1}$, which is $\approx 16 \%$ of its Eddington luminosity (Section 5.1 ).

A dynamical black hole model for the system was presented in pioneering work by Hutchings et al. (1983, 1987). Recently, we have greatly improved the dynamical model of the system (Orosz et al. 2009). The results of most relevance to this paper are our determinations of the black hole mass $M=10.91 \pm 1.54$ $M_{\odot}$ and the orbital inclination angle $i=36.38 \pm 2.02$. The distance is likewise important here; we adopt a distance modulus of $18.41 \pm 0.10 \mathrm{mag}$, which corresponds to $D=48.10 \pm 2.22$ kpc (Orosz et al. 2007, 2009). Accurate values of $M, i$, and $D$ such as these are crucial for the determination of black hole spin by the X-ray continuum-fitting method that we employ (Zhang et al. 1997; Li et al. 2005).

To date, our group has published spin estimates for four stellar-mass black holes using the X-ray continuum-fitting method: GRO J1655-40, $a_{*}=0.65-0.75$, and 4U 1543-47, $a_{*}=0.75-0.85$ (Shafee et al. 2006); GRS 1915+105, $a_{*}=$ 0.98-1.0 (McClintock et al. 2006); and M33 X-7, $a_{*}=$ $0.77 \pm 0.05$ (Liu et al. 2008). The dimensionless spin parameter $a_{*} \equiv c J / G M^{2}$, where $M$ and $J$ are the mass and angular momentum of the black hole; the value $a_{*}=0$ corresponds to a Schwarzschild black hole and $a_{*}=1$ to an extreme Kerr hole. In this paper, we show that LMC X-1 harbors a rapidly spinning black hole. Estimates of the spins of stellar-mass black holes are also being obtained by modeling the profile of the
Fe K line (see Miller et al. 2002, 2004, 2009, and references therein).

In all of our previous work cited above, we have exclusively used thermal dominant (TD) spectral data in deriving our estimates of black hole spin (McClintock \& Remillard 2006; Remillard \& McClintock 2006). The definition of the TD state is largely based on a spectral analysis that makes use of the venerable multitemperature and nonrelativistic disk model known as DISKBB in XSPEC (Mitsuda et al. 1984; Makishima et al. 1986) plus the standard power-law component POWERLAw (Tanaka \& Lewin 1995; McClintock \& Remillard 2006; Remillard \& McClintock 2006); XSPEC is a widely used Xray spectral fitting package (Arnaud 1996). The key feature of the TD state is the strong presence of a soft $(k T \sim 1 \mathrm{keV})$ blackbody-like component of emission that arises in the innermost region of the accretion disk. The TD state is defined by three criteria: (1) the "disk fraction (DF)," the fraction of the total $2-20 \mathrm{keV}$ unabsorbed flux in the thermal component, is $>75 \%$; (2) the rms power in the power density spectrum integrated from 0.1 to $10 \mathrm{~Hz}$ is $<0.075$; and (3) QPOs are absent or very weak (see Table 2 and the text in Remillard \& McClintock 2006).

Following the selection of the TD spectral data, we determine the spin parameter $a_{*}$ using our relativistic disk model KERRBB2 (McClintock et al. 2006). This model incorporates a look-up table for the spectral hardening factor $f$ and returns two fit parameters, the spin $a_{*}$ and the mass accretion rate $\dot{M}$. In addition to the thermal disk spectrum, there is also present a nonthermal tail component of emission, which contributes a few percent of the $1-10 \mathrm{keV}$ flux. This tail component is widely believed to originate from Compton upscattering of the blackbody seed photons in a hot corona (McClintock \& Remillard 2006). Source spectra obtained in the TD state are minimally corrupted by the uncertain effects of Comptonization, and they are ideal for the determination of spin via the continuum-fitting method. 
In earlier work (see especially Section 4.2 in McClintock et al. 2006), we have been hampered by the lack of an appropriate model of the tail component of emission, which we have modeled as a standard power law (POWERLAW in XSPEC) or by using one of the existing Comptonization models (e.g., COMPTT). We found both approaches unsatisfactory because the power law diverges from realistic Comptonization models at low energies (see Section 4.2) while the existing Comptonization models have more parameters than can be determined. Very recently, we have developed a simple two-parameter Comptonization model in order to treat this tail component (Steiner et al. 2009; Section 4.2). The model, which is named SIMPL, has been implemented in XSPEC. It is a convolution model that redirects a fraction of the blackbody seed photons into a power law. The benefits of using SIMPL to model the tail component are that it eliminates the unphysical divergence of a standard power law at low energies while introducing just two parameters into the fit. Its two parameters are the scattered fraction $f_{\mathrm{SC}}$, the fraction of the seed photons that are scattered into a power-law tail component, and a photon index $\Gamma$. An illustration of the difference between the models POWERLAW and SIMPL and a discussion of the benefits of using the latter model are given in Section 4.2.

In this paper, we focus on 53 RXTE spectra obtained using the Proportional Counter Array (PCA). These data are central because they are abundant, and especially because they allow one to select data via the rms variability criterion (Remillard \& McClintock 2006), which we show is important for the reliable determination of the spin of LMC X-1. LMC X-1 was also observed in single observations using XMM-Newton, BeppoSAX, BBXRT, and ASCA, and on six occasions by Ginga. In Appendix A, we analyze the spectral data obtained by these five missions and find values of spin that confirm our principal RXTE PCA results.

This paper is organized as follows. In Section 2, we discuss the selection of our gross sample of 53 RXTE spectra and their reduction. In Section 3, we present the results of a preliminary, nonrelativistic data analysis and select a final sample of 18 spectra for analysis. In Section 4, we discuss our relativistic data analysis with emphasis on our first use of the Comptonization model SIMPL. We present our results in Section 5, a general discussion in Section 6, and our conclusions in Section 7.

\section{DATA SELECTION AND REDUCTION}

As noted in Section 1, the data of central importance in this paper were obtained using the large-area PCA detector onboard RXTE (Swank 1999). We consider 53 of the total of 55 PCA pointed observations that have been performed during the course of the mission; these 53 observations are identified and characterized in Table 1. We disregard two observations that were obtained very early in the mission (1996 February 10 and 1996 March 8) because the detector response is poorly known during that period, and the PCA gain was higher than at later times. The RXTE PCA data were downloaded from NASA's High Energy Astrophysics Science Archive Research Center (HEASARC).

In our analysis, we only include pulse-height spectra from PCU-2 because fits to the power-law spectrum of the Crab show that this is the best calibrated of the five proportional counter units (PCUs) that comprise the PCA. Data reduction tools from HEASOFT version 5.2 were used to screen the event files and spectra. Data were taken in the "Standard 2 mode," which provides coverage of the PCA bandpass every $16 \mathrm{~s}$. Data from all Xe gas layers of PCU-2 were combined to make the spectra. Background spectra were derived using the tool pcabackest and the latest "faint source" background model and were then subtracted from the total spectra. Redistribution matrix files and ancillary response files were freshly generated individually for each PCU layer and combined into a single response file using the tool pcarsp. In fitting each of the pulseheight spectra (Sections 3 and 4), we used response files that were targeted to the time of each observation of LMC X-1.

Following the methods spelled out in Section 3 in McClintock et al. (2006), for the 53 observations of the source we individually (1) added the customary systematic error of $1 \%$ to all the PCA energy channels; (2) corrected the PCA count rates for dead time; and (3) corrected the effective area of the PCA using for each observation of LMC X-1 the proximate and dead-time corrected observation of the Crab Nebula (Toor \& Seward 1974). The dead-time correction factor for LMC X-1 is $\approx 1.021$. The effective-area correction factor is $\approx 1.076$, which requires us to apply a typical downward correction to the fluxes of $\approx 0.930$. The net effect of the dead-time and effective-area corrections was to reduce the LMC X-1 fluxes that we obtained from the analysis of each of our 53 spectra by $\approx 5 \%$.

\section{PRELIMINARY DATA ANALYSIS}

All of the data analysis and model fitting throughout this paper was performed using XSPEC version 12.4 (Arnaud 1996). In our two earlier papers that rely primarily on RXTE data to estimate the spins of three black holes (Shafee et al. 2006; McClintock et al.2006), for each source we performed a preliminary analysis using the nonrelativistic disk model DISKBB plus the standard power law POWERLAW for three reasons: (1) to select the TD data (Remillard \& McClintock 2006), (2) to explore the data using this time-tested model, and (3) to compare our results with published results.

For LMC X-1, we have likewise performed a nonrelativistic analysis using this conventional model, which in XSPEC notation is expressed as PHABS(DISKBB+POWERLAW) (Remillard \& McClintock 2006), where PHABs is a widely used model of lowenergy photoelectric absorption. The spectra were fitted over the energy range $2.5-20.0 \mathrm{keV}$, and the XSPEC command "energies" was invoked to accommodate the broader energy range required by the convolution model simPL. The hydrogen column density was fixed at $N_{\mathrm{H}}=4.6 \times 10^{21} \mathrm{~cm}^{-2}$, as determined by an analysis of Chandra HETG grating data (Cui et al. 2002; Orosz et al. 2009). The PCA fits are quite insensitive to the choice of the lower cutoff energy because the low-energy absorption is $\leqslant 10 \%$ above $2.5 \mathrm{keV}$. We obtained good fits to this basic model for all 53 spectra without needing to add any of the customary additional components (e.g., a Gaussian line or a broad Fe absorption edge). The fit results, along with some observational data (e.g., exposure times and count rates), are summarized in Table 1 . The two pairs of parameters returned by the fit are the two Dіsквв parameters, the temperature at the inner edge of the accretion disk $T_{\text {in }}$ and a normalization constant $K$ (Columns 7 and 8), and the two POWERLAw parameters, the photon index $\Gamma$ and a normalization constant (Columns 9 and 10).

Columns 2 and 3 in Table 1 identify the observations by calendar date and the midpoint time of the observation. Columns 4 and 5 give the total exposure time and the total source count rate. The rms power in Column 6, which is a timing parameter, is of special interest, and we return to it after commenting on the contents of the remaining columns in the table. The four spectral parameters described in the preceding paragraph are listed in Columns 7-10. The DF as defined in Section 1, which 
Table 1

Preliminary Results for LMC X-1 RXTE Data Using PHABS(DISKBB+POWERLAW) ${ }^{\text {a }}$

\begin{tabular}{|c|c|c|c|c|c|c|c|c|c|c|c|c|}
\hline $\begin{array}{l}\text { Obs. } \\
\text { No. } \\
\text { (1) } \\
\end{array}$ & $\begin{array}{c}\text { UT } \\
\text { (yyyy-mm-dd) } \\
(2) \\
\end{array}$ & $\mathrm{MJD}^{\mathrm{c}}$ & $\begin{array}{l}T_{\text {obs }} \\
(\mathrm{s}) \\
(4)\end{array}$ & $\begin{array}{c}\text { Count Rate } \\
\left(\text { counts s}^{-1}\right) \\
(5)\end{array}$ & $\begin{array}{c}\mathrm{rms} \\
\left(10^{-2}\right)^{\mathrm{d}} \\
(6)\end{array}$ & $\begin{array}{c}T_{\text {in }} \\
(\mathrm{keV}) \\
(7)\end{array}$ & (8) & (9) & $\begin{array}{l}\text { Power-law } \\
\text { Norm } \\
(10)\end{array}$ & $\begin{array}{l}\mathrm{DF}^{\mathrm{g}} \\
(\%) \\
(11) \\
\end{array}$ & State $^{\mathrm{h}}$ & $\chi_{v}^{2} / \mathrm{dof}$ \\
\hline 1 & 1996-06-09 & 50243.888 & 4960 & $1.27 \pm 0.16$ & $7.43 \pm 0.64$ & $0.864 \pm 0.008$ & $52.73 \pm 4.14$ & $3.06 \pm 0.14$ & $0.20 \pm 0.06$ & 64.45 & PL:TD & $93 / 43$ \\
\hline 2 & & & 4960 & & & & & & & & & $62 / 43$ \\
\hline 3 & & & 73168 & & & & & & & & & \\
\hline 4 & & & & & & & & & & & & $.05 / 43$ \\
\hline 5 & & & & & & & & & & & & $.74 / 43$ \\
\hline 6 & & & & & & & & $28 \pm 0.10$ & & & & $89 / 43$ \\
\hline 7 & & & & & $7.15 \pm 0.42$ & & & $19 \pm 0.12$ & & & & $0.72 / 43$ \\
\hline 8 & & & 4400 & & $6.09 \pm 0$ & & & $3.25 \pm 0.13$ & & & & $0.66 / 43$ \\
\hline 9 & & & 2768 & & & & & & & & & $0.81 / 43$ \\
\hline 10 & & & 4240 & & $5.74 \pm$ & & & $3.28 \pm$ & & & & $0.70 / 42$ \\
\hline 11 & & 50680.829 & 9056 & 15 & $7.04 \pm$ & & & & & & & $0.79 / 42$ \\
\hline 12 & & & 9456 & & & & & & & & & $0.68 / 42$ \\
\hline 13 & & & 80 & & & & & & & & & $.71 / 42$ \\
\hline 14 & & & 16 & & & & & & & & & $52 / 42$ \\
\hline 15 & & & 60 & & & & & & & & & $84 / 42$ \\
\hline 16 & & & 1248 & & & & & & & & & $.94 / 42$ \\
\hline 17 & & & & & & & & & & & & $.62 / 42$ \\
\hline 18 & 200 & 5301 & 9856 & 28.7 & $6.61 \pm$ & 0.89 & & $2.42 \pm$ & & 85.65 & TD & $0.80 / 37$ \\
\hline 19 & & & & & & & & & & & & \\
\hline 20 & & & & & & & & & & & & $79 / 43$ \\
\hline 21 & & & & & & & & & & & & $1.07 / 43$ \\
\hline 22 & & & & & & & & & & & & $1.42 / 43$ \\
\hline 23 & & & 4832 & & & & & & & & & $0.95 / 43$ \\
\hline 24 & & & & & & & & & & & & $1.06 / 43$ \\
\hline 25 & & & & & & & & & & & & \\
\hline 26 & & & 9664 & & & & & 10 & & & TD & $1.40 / 43$ \\
\hline 27 & & & & & & & & & & & & $4 / 43$ \\
\hline 28 & & & & & & & & & & & & $76 / 43$ \\
\hline 29 & & & & & & & & & & & & $99 / 42$ \\
\hline 30 & & & & & & & & & & & & $24 / 42$ \\
\hline 31 & & & & & & & & & & & & 142 \\
\hline 32 & & & & & & & & & & & & \\
\hline 33 & & & & & & & & & & & & $0.99 / 42$ \\
\hline 34 & & & & & & & & & & & & $1.27 / 42$ \\
\hline 35 & & & & & & & & & & & TD & $1.01 / 42$ \\
\hline 36 & & & & & & & & & & & & $1.23 / 42$ \\
\hline 37 & & & & & & & & & & & & $0.86 / 42$ \\
\hline 38 & & & & & & & & & & & $: \mathrm{TD}$ & $0.67 / 42$ \\
\hline & & & & & & & & & & & & $0.89 / 42$ \\
\hline & & & & & & & & & & & & $82 / 42$ \\
\hline & & & & & & & & & & & TD & $1 / 42$ \\
\hline 42 & & & & & & & & & & & & \\
\hline 43 & & & & & & & & & & & & $0.98 / 42$ \\
\hline 44 & & & 8688 & & & & & & $0.22 \pm 0.06$ & 62.82 & SPL:TD & $1.00 / 37$ \\
\hline 45 & 04-01-08 & 53012.749 & 5360 & & $7.83 \pm 0.66$ & $0.973 \pm 0.011$ & $28.62 \pm 3.04$ & $3.16 \pm 0.16$ & $0.23 \pm 0.09$ & 65.01 & SPL:TD & $0.66 / 37$ \\
\hline 46 & 04-01-09 & 53013.166 & 9264 & $31.20 \pm 0.13$ & $8.9 \pm 0.54$ & $0.977 \pm 0.010$ & $25.97 \pm 2.45$ & $3.17 \pm 0.11$ & $0.29 \pm 0.07$ & 58.06 & SPL:TD & $1.05 / 37$ \\
\hline 47 & & & & & $9 \pm 0$ & & $51.84 \pm 4.36$ & & & & L:TD & $0.72 / 37$ \\
\hline 48 & & & 10160 & & $8.61 \pm$ & & $33.78 \pm 2$ & $3.12 \pm$ & $0.23 \pm 0$ & 64.73 & SPL:TD & $1.15 / 37$ \\
\hline 49 & & & & & & & & & & & & $0.59 / 37$ \\
\hline & & & & & & & & & & & & \\
\hline 51 & & & & & & & & & & & & $0.74 / 37$ \\
\hline 52 & & & 10704 & & 1 & & & & & & SPL:TD & $0.85 / 37$ \\
\hline 53 & 2004-01-12 & 53016.822 & 4720 & $31.45 \pm 0.17$ & $11.74 \pm 0.59$ & $1.170 \pm 0.029$ & $8.28 \pm 1.47$ & $3.22 \pm 0.09$ & $0.42 \pm 0.08$ & 44.71 & SPL & $0.70 / 37$ \\
\hline
\end{tabular}

Notes.

${ }^{\text {a }}$ Column density fixed at $N_{\mathrm{H}}=4.6 \times 10^{21} \mathrm{~cm}^{-2}$

${ }^{\mathrm{b}}$ The first 18 spectra, which meet the TD criterion rms $<0.075$, comprise our favored sample of gold/silver spectra.

${ }^{\mathrm{c}}$ Midpoint time of observation. MJD $=\mathrm{JD}-2,400,000.5$.

${ }^{\mathrm{d}}$ Total rms power integrated over $0.1-10 \mathrm{~Hz}$ in the power density spectrum $(2-30 \mathrm{keV})$.

e Normalization constant of the thermal component: $K \propto R_{\text {in }}^{2}$, where $R_{\text {in }}$ is the inner-disk radius.

${ }^{\mathrm{f}}$ Photon index of the power-law component.

${ }^{g}$ Disk fraction defined over the energy range 2-20 keV.

${ }^{\mathrm{h}}$ See Section 1 and the text in Remillard \& McClintock (2006) for state definitions. 
Table 2

Primary Results for LMC X-1 RXTE Data Using PHABS(SIMPL $\otimes$ KERRBB2) ${ }^{\mathrm{a}}$

\begin{tabular}{|c|c|c|c|c|c|c|c|c|c|}
\hline No. & MJD & $a_{*}$ & $\dot{M}^{\mathrm{b}}$ & $f$ & $\Gamma$ & $f_{\mathrm{SC}}$ & $\operatorname{NDF}(\%)$ & $\chi_{v}^{2} / \mathrm{dof}^{\mathrm{c}}$ & $L / L_{\text {edd }}$ \\
\hline 1 & 50243.888 & $0.933 \pm 0.007$ & $1.256 \pm 0.040$ & 1.557 & 2.5 & $0.049 \pm 0.002$ & 85.87 & $0.89 / 44$ & 0.145 \\
\hline 2 & 50296.917 & $0.884 \pm 0.008$ & $1.679 \pm 0.047$ & 1.551 & 2.5 & $0.044 \pm 0.001$ & 86.84 & $0.56 / 44$ & 0.164 \\
\hline 3 & 50423.854 & $0.962 \pm 0.003$ & $1.183 \pm 0.021$ & 1.567 & 2.5 & $0.035 \pm 0.001$ & 90.29 & $1.37 / 44$ & 0.158 \\
\hline 4 & 50516.454 & $0.949 \pm 0.005$ & $1.262 \pm 0.032$ & 1.573 & 2.5 & $0.038 \pm 0.001$ & 89.36 & $1.04 / 44$ & 0.157 \\
\hline 5 & 50528.108 & $0.944 \pm 0.005$ & $1.333 \pm 0.031$ & 1.574 & 2.5 & $0.032 \pm 0.001$ & 90.93 & $0.80 / 44$ & 0.162 \\
\hline 6 & 50554.291 & $0.928 \pm 0.005$ & $1.431 \pm 0.032$ & 1.566 & 2.5 & $0.037 \pm 0.001$ & 89.35 & $0.98 / 44$ & 0.161 \\
\hline 7 & 50575.107 & $0.938 \pm 0.004$ & $1.379 \pm 0.031$ & 1.573 & 2.5 & $0.036 \pm 0.001$ & 89.84 & $0.74 / 44$ & 0.162 \\
\hline 8 & 50596.944 & $0.952 \pm 0.006$ & $1.193 \pm 0.040$ & 1.569 & 2.5 & $0.043 \pm 0.002$ & 87.98 & $0.72 / 44$ & 0.151 \\
\hline 9 & 50597.047 & $0.939 \pm 0.007$ & $1.260 \pm 0.045$ & 1.563 & 2.5 & $0.033 \pm 0.002$ & 90.26 & $0.78 / 44$ & 0.150 \\
\hline 10 & 50638.653 & $0.916 \pm 0.009$ & $1.437 \pm 0.054$ & 1.550 & 2.5 & $0.033 \pm 0.002$ & 89.98 & $0.66 / 43$ & 0.155 \\
\hline 11 & 50680.829 & $0.945 \pm 0.005$ & $1.401 \pm 0.037$ & 1.572 & 2.5 & $0.033 \pm 0.001$ & 90.83 & $0.75 / 43$ & 0.170 \\
\hline $12^{\mathrm{d}}$ & 50703.106 & $0.909 \pm 0.007$ & $1.594 \pm 0.043$ & 1.551 & 2.5 & $0.032 \pm 0.001$ & 90.44 & $0.58 / 43$ & 0.168 \\
\hline 13 & 50710.736 & $0.966 \pm 0.005$ & $1.160 \pm 0.035$ & 1.554 & 2.5 & $0.042 \pm 0.001$ & 88.54 & $0.67 / 43$ & 0.159 \\
\hline 14 & 50794.380 & $0.926 \pm 0.006$ & $1.499 \pm 0.039$ & 1.561 & 2.5 & $0.028 \pm 0.001$ & 91.79 & $0.57 / 43$ & 0.168 \\
\hline 15 & 50884.606 & $0.965 \pm 0.004$ & $1.257 \pm 0.036$ & 1.569 & 2.5 & $0.036 \pm 0.001$ & 90.37 & $0.83 / 43$ & 0.171 \\
\hline 16 & 50939.252 & $0.947 \pm 0.005$ & $1.295 \pm 0.036$ & 1.562 & 2.5 & $0.033 \pm 0.001$ & 90.56 & $0.84 / 43$ & 0.160 \\
\hline $17^{\mathrm{d}}$ & 51014.014 & $0.936 \pm 0.005$ & $1.374 \pm 0.037$ & 1.561 & 2.5 & $0.029 \pm 0.001$ & 91.50 & $0.49 / 43$ & 0.161 \\
\hline $18^{\mathrm{d}}$ & 53011.559 & $0.940 \pm 0.005$ & $1.273 \pm 0.036$ & 1.551 & 2.5 & $0.030 \pm 0.001$ & 91.03 & $0.76 / 38$ & 0.151 \\
\hline 19 & & $0.938 \pm 0.020^{\mathrm{e}}$ & $1.348 \pm 0.137$ & $1.562 \pm 0.008$ & 2.5 & $0.036 \pm 0.005$ & $89.76 \pm 1.53$ & $0.78 \pm 0.20$ & $0.160 \pm 0.007$ \\
\hline
\end{tabular}

Notes.

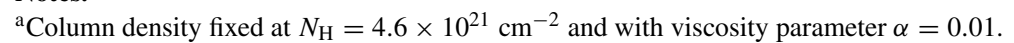

${ }^{\mathrm{b}}$ The mass accretion rate is in units of $10^{18} \mathrm{~g} \mathrm{~s}^{-1}$.

$\mathrm{c}$ The number of degrees of freedom (dof) in Table 1 is one less because here $\Gamma$ is fixed at 2.5.

${ }^{\mathrm{d}}$ The three strictly TD-state spectra; their average spin parameter is $a_{*}=0.928 \pm 0.014$.

'The value of the spin parameter was computed using the one-sided Green's function simPL-I (Section 4.2). The two-sided Green's function gives an essentially identical result with a mean spin parameter $a_{*}=0.937 \pm 0.020$. If one includes a Gaussian emission line in the fit to all 18 spectra, PHABS(SIMPL $\otimes\left(\right.$ KERRBB2+GAUSSIAN)), the average spin parameter decreases slightly by 0.6 standard deviations to $a_{*}=0.922 \pm 0.021$ (Section 5.2$)$.

characterizes the relative strength of the thermal component, is given in Column 11. The state of the source (see Table 2 and the text in Remillard \& McClintock 2006) is given in Column 12 , and the reduced $\chi^{2}$ and the number of degrees of freedom are given in the final column.

An inspection of Column 12 in Table 1 shows that only three out of our 53 spectra are classified as TD spectra (Nos. 12, 17, and 18). ${ }^{7}$ We consider our results for these spectra to be of the highest reliability, and we therefore refer to them as our "gold" spectra. However, this sample is small, and so we consider a second tier of quality, namely those spectra that fail to meet the TD criterion that the disk fraction exceed $75 \%$, but which do meet the TD criterion that the rms power is $<0.075$ (Section 1). These are our 15 "silver" spectra, and they correspond to the following observations listed in Table 1: Obs. Nos. 1-11 and 13-16. Our adopted value of the spin parameter (Section 5.1) is based on the results for all 18 gold and silver spectra; in Section 6, we show that our spin results for both types of spectra are in excellent agreement.

Meanwhile, the 35 spectra in the lower part of Table 1 (Nos. 19-53) fail to meet the first two TD selection criteria. We refer to these as our bronze spectra, and we do not include these data in determining our adopted value of $a_{*}$. However, in Section 6 we show that these bronze spectra yield a value of $a_{*}$ that is consistent with that obtained from the gold/silver spectra, although this value is less well constrained. Throughout this paper we focus our attention on our selected sample of 18 gold/ silver spectra, all of which satisfy the temporal TD criterion that the rms power is $<0.075$.

\footnotetext{
7 QPOs were absent in these spectra and all 53 RXTE PCA spectra, although they have been reported for LMC X-1 in Ginga observations by Ebisawa et al. (1989) and in BeppoSAX observations by Dal Fiume et al. (2001).
}

\section{DATA ANALYSIS}

The summary in Table 1 of our preliminary analysis results indicates the challenges faced in determining the spin of LMC $\mathrm{X}-1$ via the continuum-fitting method. All but three of the spectra fail to meet the most fundamental criterion that defines the TD state, namely that the unabsorbed thermal component comprise at least $75 \%$ of the $2-20 \mathrm{keV}$ flux (Column 11). A related problem is the steepness of the power-law component $(\Gamma \gtrsim 3$; Column 9), which causes the power-law flux to rival or exceed the thermal flux at low energies, a situation that we regard as unphysical for a Comptonization model (Steiner et al. 2009). Finally, 35 of the 53 spectra fail to meet a second basic criterion of the TD state: rms continuum power $<0.075$ (Section 1; Table 1).

In this section, we describe in turn our workhorse relativistic disk model KERRBB2 and the Comptonization model SIMPL. We then summarize the complete set of parameters for the composite model that we employ and close by discussing the necessity of fixing the power-law index. Meanwhile, in Appendix B, we consider and reject the possibility that the spectrum of LMC $\mathrm{X}-1$ is contaminated by flux from the nearby X-ray pulsar PSR B0540-69.

\subsection{The Relativistic Accretion Disk Model: KERRBB2}

Our model of a thin accretion disk around a Kerr black hole ( $\mathrm{Li}$ et al. 2005) includes all relativistic effects such as frame dragging, Doppler boosting, gravitational redshift, and light bending. It also includes self-irradiation of the disk ("returning radiation") and the effects of limb darkening. The effects of spectral hardening are incorporated into the basic model KERRBB via a pair of look-up tables for the spectral hardening factor $f$ corresponding to two representative values 
of the viscosity parameter: $\alpha=0.01$ and 0.1 (see Section 5.3). The entries in this table were computed using a second relativistic disk model BHSPEC (Davis et al. 2005; Davis \& Hubeny 2006). We refer to the model KERRBB plus this table/ subroutine as KERRBB2, which is the accretion-disk model that we use hereafter in this paper. The model KERRBB2 has just two fit parameters, namely the black hole spin $a_{*}$ and the mass accretion rate $\dot{M}$, or equivalently, $a_{*}$ and the Eddingtonscaled bolometric luminosity, $L_{\text {bol }}\left(a_{*}, M\right) / L_{\text {Edd }}$, where $L_{\text {Edd }}=$ $1.3 \times 10^{38}\left(M / M_{\odot}\right) \mathrm{erg} \mathrm{s}^{-1}$ (Shapiro \& Teukolsky 1983). For a detailed discussion of KERRBB2 and its application, see Section 4 in McClintock et al. (2006).

Throughout this paper, we present detailed results only for the case $\alpha=0.01$. However, we have computed all of the same results for the case $\alpha=0.1$ as well. As we show in Section 5.3 , the choice of the higher value of $\alpha$ results in a slightly lower value of $a_{*}$. Importantly, our final adopted value for the spin parameter $a_{*}$ and its error takes into account the inherent uncertainty in $\alpha$ (Sections 5.3 and 5.6).

\subsection{The Comptonization Model: SIMPL}

In this paper, we make the first application of the Comptonization model SIMPL (Steiner et al. 2009) in the determination of the spin of a black hole. The model was introduced in Section 1 where our motivations for developing it and its importance in this present work are discussed. The model is fully described in Steiner et al. (2009) where it is applied to data and its performance is shown to compare favorably to such physical models of Comptonization as COMPTT and COMPBB. The model SIMPL (SIMple Power Law) functions as a convolution that converts a fraction $f_{\mathrm{SC}}$ of seed photons into a power law with photon index $\Gamma$. There are two implementations in XSPEC: SIMPL-I, which uses a one-sided Green's function and corresponds to upscattering only, and SIMPL-2, which uses a classical two-sided Green's function (Sunyaev \& Titarchuk 1980) and corresponds to both up and downscattering of photons. SIMPL-I is a "bare-bones" and computationally efficient implementation. In our application, the two models give nearly identical results for the spin parameter. Generically, throughout this paper we refer to both versions of the code as SIMPL; however, all of the analysis in this paper is done using SIMPL-I.

Because SIMPL is a convolution model it ties the Comptonized component directly to the energy distribution of the input photons, which in the application at hand is specified by our disk model KERRBB2. The most important feature of SIMPL is that it produces a power-law tail at energies higher than those of the thermal seed photons, but the power law does not extend to lower energies, which is a property shared by all physical Comptonization models (e.g., COMPTT). Thus, the crucial difference between SIMPL and the standard power law is that SIMPL cuts off in a physically natural way, whereas the standard power law diverges at low energy. At the same time, SIMPL is an empirical model that, while mimicking the mechanics of scattering, makes as few assumptions as possible. In particular, the model has only two free parameters, as few as the standard power law.

Figure 1 starkly illustrates the quite different models one obtains employing POWERLAW versus SIMPL. Shown are fits to one of our 18 selected spectra using the models PHABS(DISKBB+POWERLAW) and PHABS(SIMPL $\otimes$ DISKBB). Contrasting behaviors between POWERLAW and SIMPL are most clearly revealed at low energies in the unabsorbed models, which are shown in the rightward pair of panels. Note how the power-law component rises without limit, whereas the SIMPL model cuts off in a physically natural way in the manner of all Comptonization models. Because POWERLAW produces higher fluxes than SIMPL at low energies, it steals flux from the thermal component and hardens it, thereby reducing its normalization constant. Furthermore, the rising power-law component yields inflated estimates of $N_{\mathrm{H}}$ relative to SIMPL and other Comptonization models. Thus, although the quality of fit using either of these two-parameter models is comparable, they can yield quite different results for both the parameters returned by the thermal component and for $N_{\mathrm{H}}$. These differences between POWERLAW and SIMPL are most pronounced when the power law is relatively steep, $\Gamma \gtrsim 3$, as in the case of LMC X-1.

When the model SIMPL is convolved with a thermal component, in addition to the observed thermal component, one also has available the seed spectrum, i.e., the thermal spectrum prior to Comptonization. Thus, there are two possible definitions of the disk fraction: the classical definition of the disk fraction DF that is based on the observed component of thermal flux (Remillard \& McClintock 2006), and a new definition based on the flux of the blackbody seed photons, i.e., the model-dependent flux that emerges from the surface of the disk ignoring the effects of the corona that dresses it. We refer to this disk fraction as the "naked disk fraction" or NDF.

The two disk fractions are simply related via the scattered fraction $f_{\mathrm{SC}}$ : DF $=\left(1-f_{\mathrm{SC}}\right) \mathrm{NDF}$. The choice of which of the two disk fractions to use depends on the purpose at hand. The disk fraction DF is currently used to classify the state of the source (Section 3), whereas the naked disk fraction NDF is useful for assessing energetics (e.g., in relating the X-ray power to the thermal energy stored in the corona). Throughout this paper, we have chosen to use the naked disk fraction and we compute it for the canonical energy interval $2-20 \mathrm{keV}$.

\subsection{Description of Model Parameters}

In all of the relativistic data analysis, we use KERRBB2 convolved with SIMPL in conjunction with PHABS, which models the low-energy absorption, i.e., our XSPEC model is PHABS(SIMPL $\otimes$ KERRBB2). We use precisely the same energy range, $2.5-20.0 \mathrm{keV}$, and fixed hydrogen column density, $N_{\mathrm{H}}=$ $4.6 \times 10^{21} \mathrm{~cm}^{-2}$, that we used in our preliminary data analysis (Section 3). The number of fit parameters is likewise the same, namely four in total: two for KERRBB2, the spin parameter $a_{*}$ and the mass accretion rate $\dot{M}$, and two for SIMPL, the power-law index $\Gamma$ and the scattered fraction $f_{\mathrm{SC}}$. The normalization constant of KERRBB2 is fixed at unity, which is appropriate when $M, i$, and $D$ are held fixed at the values quoted in Section 1. For KERRBB2 we also include the effects of limb darkening (lflag=1) and returning radiation ( $\mathrm{rflag}=1)$, and the torque at the inner boundary of the accretion disk is set at zero $(\eta=0$; Shafee et al. 2008a, 2008b). In the case of the Comptonization model SIMPL, we set switch UpScOnly=1, i.e., we use the SIMPL-I version of the code, which corresponds to upscattering only (Section 4.2).

\subsection{Fixing the Power-law Index $\Gamma$}

We further reduced the number of free fit parameters from four to three by fixing the spectral index $\Gamma$. We did this because the power-law component is not well constrained, as we now explain. LMC X-1 is classified as a faint source for the PCA, i.e., its total count rate is $<40$ counts $\mathrm{cm}^{-2} \mathrm{~s}^{-1}$ per PCU (Table 1). The PCA background rate is determined using a model, in this case the "faint source background model," which is keyed to 


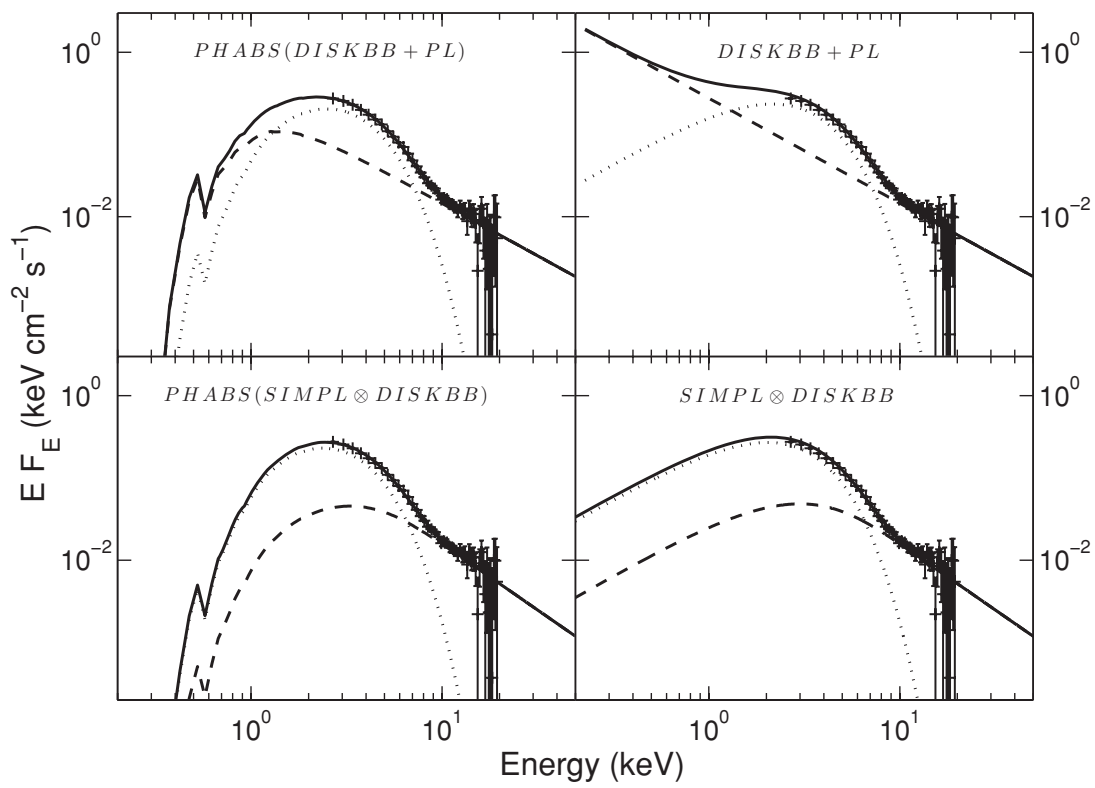

Figure 1. POWERLAW vs. SIMPL. The left pair of panels show unfolded spectral fits to a PCA observation of LMC X-1 (No. 5 in Table 1) and the pair on the right show the corresponding unabsorbed models. All of the fits were performed using DISKBB in conjunction with POWERLAW (top panels) and sIMPL (lower panels). The composite model is represented by a solid line, the emergent disk component by a dotted line, and the Comptonization component by a dashed line. Note the strikingly different behaviors of this latter component in the unabsorbed models.

the count rate of a charged-particle detector aboard the RXTE spacecraft (Jahoda et al. 2006).

We find that there are quite significant systematic uncertainties in the background count-rate model. This is clearly shown by computing the count rates for the 18 gold/silver PHA spectra for the energy range $20-40 \mathrm{keV}$, where our models predict that the source count rate is completely negligible relative to the background rate. We first note that these background rates indicate that the model background has been overestimated because for 14 of the 18 spectra the rates are negative. More telling is the fact that these 18 background rates deviate from the expected value of zero count rate on average by $4.6 \sigma$ (std. dev.; $N=18$ ). These significant deviations show that the background model provides a poor approximation to the true background rate.

We now consider the net source count rates for the 18 gold/ silver PHA spectra for the energy range 10-20 keV, which is crucial for the determination of the power-law parameters (while being completely unaffected by the $\sim 1 \mathrm{keV}$ thermal component). Using the $20-40 \mathrm{keV}$ photon rates as a proxy for the 10-20 keV background rates, we find for half of the 18 observations (Nos. 1-3, 7-9, and 15-17 in Table 1) that the background rates are fully one-fifth of the source count rates. Subtracting this relatively large and uncertain background rate from the total count rate significantly corrupts the source spectrum. Thus, for this faint source we conclude that we cannot adequately constrain the power-law index, and we therefore fix it at the reasonable nominal value of $\Gamma=2.5$ (Remillard \& McClintock 2006). The good news is that our final value of the spin parameter $a_{*}$ is very insensitive to this choice of $\Gamma$, as we show in Section 5.2.

\section{RESULTS}

We first present our featured results for the 18 gold/silver spectra in Section 5.1. In Section 5.2, these results are compared to those obtained using the standard power law in place of SIMPL and also to those obtained using different fixed values of $\Gamma$. In Section 5.3, we examine the sensitivity of our results to varying the metallicity $Z$ and the viscosity parameter $\alpha$. In Section 5.4, we consider all $53 \mathrm{gold} / \mathrm{silver} /$ bronze spectra and examine the correlations between selected pairs of the fit parameters. Then, in Section 5.5, we show how the scattered fraction and the naked disk fraction depend systematically on the rms power, and we explore the usefulness of the scattered fraction $f_{\mathrm{SC}}$ in selecting data. Finally, in Section 5.6 we derive the error in the spin parameter $a_{*}$ that includes all sources of observational uncertainty, as well as the uncertainties in the model parameters. In Sections 5.1-5.5, the parameters $M, i$, and $D$ are held fixed at their best central values (see Section 1), and they are allowed to vary only in Section 5.6.

\subsection{Primary Results}

Our featured result for the spin parameter is given in the bottom line of Table 2: $a_{*}=0.938 \pm 0.020$ (std. dev.; $N=18$ ). Note that this value, which is based on all 18 gold/silver spectra, is entirely consistent with the value obtained by analyzing just the three gold TD spectra $\left(a_{*}=0.928 \pm 0.014\right.$; see footnote $\mathrm{c}$ in Table 2).

Table 2 contains all of our fit results for the 18 gold/silver spectra obtained using our relativistic disk model convolved with our Comptonization model. The uncertainties for the mean values given in the bottom line are the standard deviation for $N=18$. From left to right, $a_{*}$ and $\dot{M}$ are the fitted values of the spin parameter and the mass accretion rate, and $f$ is the interpolated value of the spectral hardening factor (Section 4.1). The two fit parameters of SIMPL follow: the photon index $\Gamma$ (which is held fixed at 2.5) and the scattered fraction $f_{\mathrm{SC}}$ (which is fitted). The next quantity is the naked disk fraction as defined for our Comptonization model (see Section 4.2). In the following column, the value of $\chi_{v}^{2}$ and the number of degrees of freedom show that the fits are all quite good with $\chi_{v}^{2}<1.4$. The final column gives the Eddington-scaled bolometric luminosity (Section 4.1) corresponding to the naked disk, which is larger than the observed disk luminosity by the factor $1 /\left(1-f_{\mathrm{SC}}\right)$, i.e., by $3 \%-5 \%$. For all the results given in Table 2 the photon index 


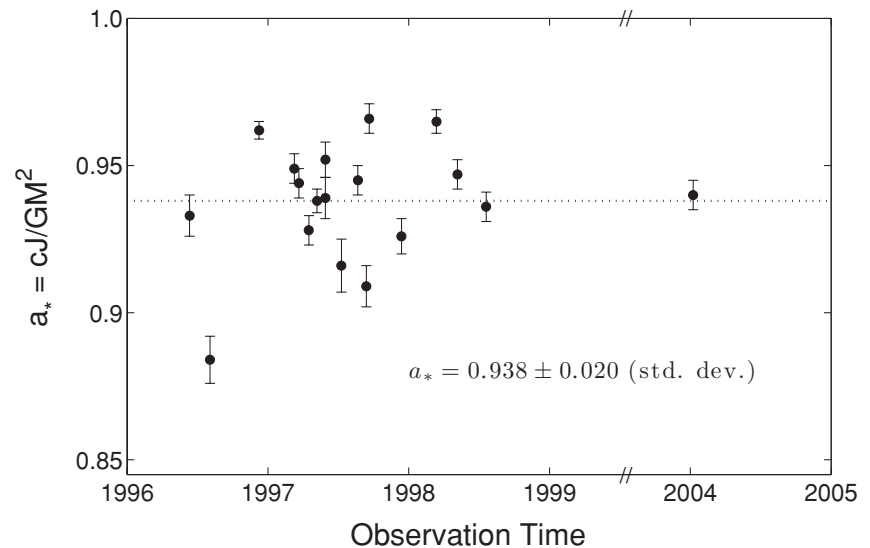

Figure 2. Spin parameter vs. observing time. The dashed line indicates the mean value of the spin parameter for the 18 gold/silver spectra. As indicated, the scatter about the mean - an effective measure of the observational uncertaintyis $\approx 2 \%$. The small error bars shown reflect only the counting-statistical errors associated with the many counts detected per observation $\left(\gtrsim 10^{5}\right.$ counts; Table 1).

was fixed at $\Gamma=2.5$ (Section 4.4), and the column density was fixed at $N_{\mathrm{H}}=4.6 \times 10^{21} \mathrm{~cm}^{-2}$ (Section 3$)$.

The luminosity is a modest fraction of the Eddington value and shows little variation over the 1996-2004 period during which these 18 spectra were obtained: $L / L_{\text {Edd }}=0.160 \pm 0.007$ (Table 2). The relative constancy of LMC X-1 is also attested to by the 12 year record of the RXTE ASM. The intensity of LMC $\mathrm{X}-1$ has been more or less steady at $\approx 20$ mcrab $(1.5-12 \mathrm{keV})$ with variations averaged over 10 day intervals of only $\approx \pm 25 \%$ (Orosz et al. 2009). In this work, as in all previous work, we select only those observations for which $L / L_{\text {Edd }}<0.3$, which corresponds to a disk that is geometrically thin at all radii, with a height-to-radius ratio less than 0.1 (McClintock et al. 2006). An inspection of the last column in Table 2 shows that all 18 gold/silver spectra easily meet this requirement.

On a timescale of many thousands of years, one fully expects the spin of the black hole to be constant, essentially unaffected by either accretion torques or any process that might extract angular momentum (King \& Kolb 1999; Liu et al. 2008). As shown in Figure 2 and as expected, the spin of LMC X-1 over the 8 year span of the RXTE observations is consistent with a constant value of $a_{*}$ to within $\approx 2 \%$ (std. dev.; $N=18$ ).

Importantly, the high spin we find using RXTE PCA data, $a_{*}=0.938 \pm 0.020$, is confirmed by X-ray missions with lowenergy response that reaches well below the $2.5 \mathrm{keV}$ cutoff of the PCA. As we show in Appendix A, when we apply the data selection criterion developed in Section $5.5\left(f_{\mathrm{SC}}<4 \%\right)$, we find values of spin using spectra obtained by the XMM-Newton, BeppoSAX, and Ginga missions that are consistent with those we obtain using our RXTE spectra.

\subsection{Results Obtained Using Other Models and Using Different Values of $\Gamma$}

In all earlier work, we have relied on the standard model PHABS(KERRBB2+POWERLAW) in deriving the spin parameter (Section 3). We found that this model was generally satisfactory (but see Section 4 in McClintock et al. 2006) because the power law was not too steep $(\Gamma \sim 2.5)$. We now apply this old model to the 18 gold/silver spectra of LMC X-1 with $\Gamma \gtrsim 3$ (Table 1) in order to see how much the steepness of the power law affects our determination of $a_{*}$. In making this comparison, we follow exactly the steps in our earlier analysis except that we model the Comptonized tail component of emission using the additive component POWERLAW rather than convolving KERRBB2 with SIMPL. For example, we fix $\Gamma=2.5$ and $N_{\mathrm{H}}=4.6 \times 10^{21} \mathrm{~cm}^{-2}$ and fit over the energy range $2.5-20.0 \mathrm{keV}$. The resultant mean fit parameters for the 18 spectra are given in line 3 of Table 3 . For ease of comparison our featured mean fit parameters, which are repeated from Table 2, are given in the first line of Table 3. Comparing lines 1 and 3, one sees that the old model yields a value of $a_{*}=0.953 \pm 0.018$, which differs from our featured value of $a_{*}=0.938 \pm 0.020$ by only 0.7 standard deviation.

How sensitive are our adopted results in Table 2 to our chosen value of $\Gamma=2.5$ (Section 4.4)? In order to answer this question, we have recomputed the results given in Table 2 with the photon index fixed at $\Gamma=2.3$ and $\Gamma=2.7$, and we find respectively, $a_{*}=0.944 \pm 0.019$ and $a_{*}=0.932 \pm 0.021$. These values differ from our adopted value of $a_{*}=0.938 \pm 0.020$ by only 0.2 standard deviations. Even if we fix the photon index at $\Gamma=3.1$, the mean value in Table 1 for the 18 gold/silver spectra (as well as for all 53 spectra), we find $a_{*}=0.918 \pm 0.024$, which differs from our adopted value by 0.6 standard deviation. This small difference is not unexpected given that so few thermal photons are scattered into the tail component, $f_{\mathrm{SC}}=0.036 \pm 0.005$ (Table 2). That $a_{*}$ is anticorrelated with $\Gamma$ is expected because a steeper power-law component steals photons from the highenergy tail of the thermal component, and the resultant softening of this component implies a lower value of spin (and vice versa).

As a final detail, we note that for just four of our 18 spectra there is clear evidence for an $\mathrm{Fe}$ emission line in the fit residuals. In deriving our primary results presented in Section 5.1, we did not include an $\mathrm{Fe}$ line because the fits are quite good without it. As a test, we have refitted all 18 spectra while including the $\mathrm{Fe}$ line, and we find a resulting mean spin parameter of $a_{*}=0.922 \pm 0.021$, which is quite consistent with our featured result above (see footnote "e" in Table 2).

\subsection{Results for Different Values of Metallicity and Viscosity Parameter}

The metallicity of the LMC, and presumably LMC X-1 as well, is $Z \approx 0.3 Z_{\odot}$ (Dopita \& Sutherland 2003), whereas our results are all given for $Z=Z_{\odot}$. How important is this

Table 3

Comparison of Fit Results for Different Models and Data Samples

\begin{tabular}{|c|c|c|c|c|c|c|}
\hline $\begin{array}{r}\text { Model } \\
\end{array}$ & $a_{*}$ & $\dot{M}$ & $\Gamma$ & $f_{\mathrm{SC}}$ & $\operatorname{NDF}(\%)$ & Comments \\
\hline PHABS(SIMPL $\otimes$ KERRBB2) ${ }^{\mathrm{a}}$ & $0.938 \pm 0.020$ & $1.348 \pm 0.137$ & 2.5 & $0.036 \pm 0.005$ & $89.76 \pm 1.53$ & 18 spectra with $\Gamma$ fixed \\
\hline 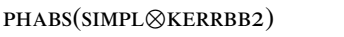 & $0.929 \pm 0.020$ & $1.388 \pm 0.136$ & $2.76 \pm 0.29$ & $0.050 \pm 0.016$ & $88.55 \pm 2.16$ & 18 spectra with $\Gamma$ free \\
\hline PHABS(KERRBB2+POWERLAW) & $0.953 \pm 0.018$ & $1.193 \pm 0.133$ & 2.5 & $\ldots$ & $84.11 \pm 2.28$ & 18 spectra with $\Gamma$ fixed \\
\hline PHABS(SIMPL $\otimes$ KERRBB2) & $0.961 \pm 0.033$ & $1.137 \pm 0.293$ & 2.5 & $0.051 \pm 0.018$ & $86.82 \pm 3.62$ & 53 spectra with $\Gamma$ fixed \\
\hline
\end{tabular}

Notes.

${ }^{\mathrm{a}} a_{*}=0.944 \pm 0.019$ for $\Gamma$ fixed at 2.3 and $a_{*}=0.932 \pm 0.021$ for $\Gamma$ fixed at 2.7 . In the extreme case of $\Gamma=3.1, a_{*}=0.918 \pm 0.024$. 
difference in metallicity? We recalculated all of the results summarized in Table 2 for the 18 gold/silver spectra using readily available BHSPEC table models that were computed for $Z=0.1 Z_{\odot}$. We found $a_{*}=0.937 \pm 0.020$ (std. dev.; $N=$ 18 ), which is almost identical to the bottom-line result quoted in Table $2\left(a_{*}=0.938 \pm 0.020\right)$. Thus, varying the metallicity has a completely negligible effect on our results.

In order to streamline the paper, throughout we quote detailed results only for one value of the viscosity parameter, $\alpha=0.01$. Presently, there is an inherent uncertainty in $\alpha$, and we direct the reader to King et al. (2007) for a more comprehensive review of numerical and observational constraints. In addition, a detailed discussion of BHSPEC's spectral dependence on $\alpha$ can be found in Done \& Davis (2008). A low value is motivated by shearing box simulations with zero net magnetic flux, which show $\alpha$ approaching zero as resolution increases (Pessah et al. 2007; Fromang \& Papaloizou 2007). The spectral models are remarkably insensitive to $\alpha$ for $\alpha \leqslant 0.01$. Therefore, results obtained for models with $\alpha=0.01$ are representative of those obtained for models with even lower values of $\alpha$.

Meanwhile, larger values of the viscosity parameter, $\alpha \sim 0.1$, are suggested by global GRMHD simulations (Hawley \& Krolik 2001; Shafee et al. 2008a) and the disk instability model (Lasota 2001). Our results for $a_{*}$ are modestly sensitive to these larger values of $\alpha$. Therefore, we have recalculated all of the results summarized in Table 2 using BHSPEC table models computed for $\alpha=0.1$. In this case, we find a slightly lower value of the spin parameter: $a_{*}=0.908 \pm 0.023$. This value is 1.0 standard deviations less than the value given in the bottom line of Table 2 for $\alpha=0.01\left(a_{*}=0.938 \pm 0.020\right)$. In deriving our final adopted value of the spin parameter $a_{*}$ and its error (Section 5.6), we take into account the uncertainty in the viscosity parameter by computing our results using both of our fiducial values of $\alpha$ : 0.01 and 0.1 .

\subsection{Graphical Presentation of Results for All 53 Spectra}

Our adopted results (Section 5.1) are based exclusively on the 18 gold/silver spectra. All of the fitting results for these spectra are given in Table 2, and histograms for the four fit parameters are shown as filled blocks in Figure 3. Although we
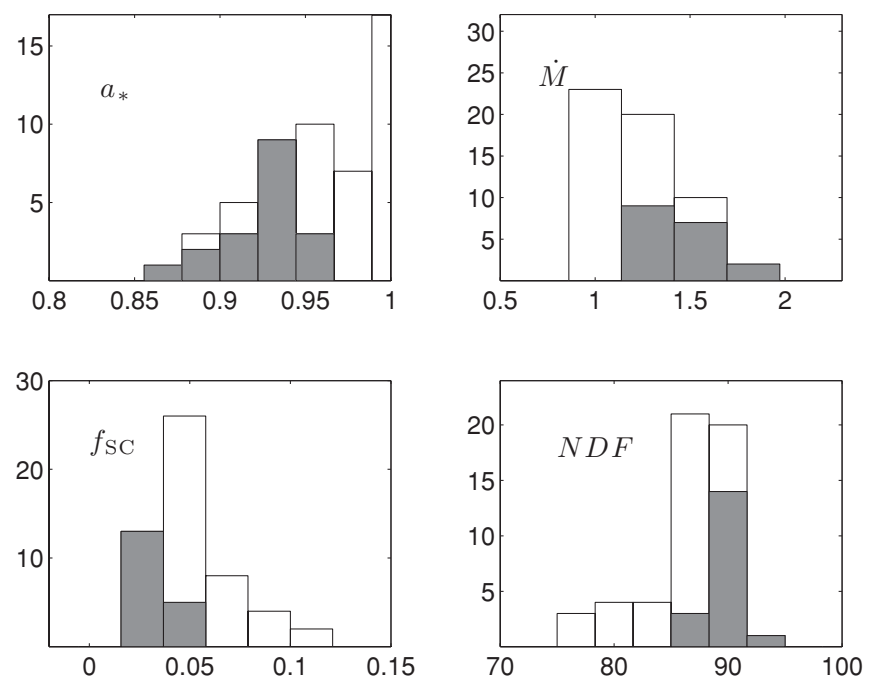

Figure 3. Histograms of the fitting parameters for our adopted model PHABS(SIMPL $\otimes$ KERRBB2) with $\Gamma$ fixed at 2.5 . The solid histograms are for the 18 gold/silver spectra and the open plus solid histograms are for all 53 gold/silver/bronze spectra. do not present a table of our results for the 35 bronze spectra (Nos. 19-53 in Table 1), we do show histograms based on the results of our analysis of these spectra as open blocks in Figure 3, which are superposed on the solid-block histograms. An inspection of the figure shows that, when compared to the 18 gold/silver spectra, the fits to all 53 spectra yield on average higher values of the spin parameter $a_{*}$, lower mass accretion rates $(\dot{M})$, more scattering of the thermal photons $\left(f_{\mathrm{SC}}\right)$, and smaller naked disk fractions (NDFs). A quantitative comparison of these differences is provided by a comparison of the results for the 18 gold/silver spectra, which are given in the top line of Table 3, with the comparable results we obtained for all 53 spectra, which are given in the bottom line of Table 3 .

Figure 4 shows correlation plots between pairs of fit parameters. The $18 \mathrm{gold} /$ silver spectra are indicated by the filled circles and the 35 bronze spectra by open circles. Figure 4(a) shows a strong correlation between $a_{*}$ and the mass accretion rate $\dot{M}$. The range of the correlation is much larger for the bronze spectra, extending to $a_{*} \approx 1$. This correlation is rather uninteresting because it simply reflects the fact that $\dot{M}$ is a normalization constant that must necessarily decrease if the measured parameter $a_{*}$ fluctuates upward (or vice versa). In Figure 4(b), we recast this correlation by plotting $a_{*}$ versus $\eta \dot{M}$, where the efficiency parameter $\eta=L_{\mathrm{bol}} / \dot{M} c^{2}$ is computed for the particular value of $a_{*}$. As shown, there is no correlation between $a_{*}$ and $\eta \dot{M}$, i.e., between $a_{*}$ and the bolometric luminosity.

Figure 4(c) shows a tight correlation between the naked disk fraction and the scattered fraction. Our adopted disk fraction (Section 4.2) is the ratio of the flux in the naked disk component $F_{\mathrm{ND}}$ to the total unabsorbed flux from 2 to $20 \mathrm{keV}$, which is the observed thermal flux plus the power-law component, $\left(1-f_{\mathrm{SC}}\right) F_{\mathrm{ND}}+F_{\mathrm{PL}}$. As $f_{\mathrm{SC}}$ increases and thermal photons are directed into the power-law tail, $F_{\mathrm{PL}}$ is expected to increase substantially because each Compton-upscattered photon is boosted in energy. Thus, as $f_{\mathrm{SC}}$ and $F_{\mathrm{PL}}$ increase, the disk fraction must decrease, as shown.

The relationship between $a_{*}$ and the scattered fraction is shown in Figure 4(d). There is no clear correlation for the 18 gold/silver spectra. However, among the 35 bronze spectra $a_{*}$ is seen to increase with scattered fraction, although the correlation is weak and there are two discrepantly low points.

\subsection{Using the Scattered Fraction to Select Data}

Figure 5 shows correlation plots of the scattered fraction and naked disk fraction versus the rms power (Section 1). The solid vertical line at rms power $=0.075$ divides the bronze data on the right from the gold/silver data on the left. These latter data are weakly correlated, showing a tendency for the scattered fraction to increase and the naked disk fraction to decrease as the rms power increases. To the right of the dividing line the sense of the correlations are the same, but the correlations steepen and become very pronounced. Of course, one expects the scattered fraction and the naked disk fraction to correlate in the opposite sense given the tight anticorrelation between these quantities that is shown in Figure 4(c).

The TD-state criterion that the rms power is $<0.075$ has played a central role in the selection of our data (Section 3). Unfortunately, the use of the rms power criterion, which requires timing data up to $\sim 10 \mathrm{~Hz}$ and is based on the particular broad energy response of the PCA, is applicable to RXTE data only. Meanwhile, the good correlation between the rms power and the scattered fraction (Figure 5) allows the latter quantity to be used in selecting data as a proxy for the rms power. (One 

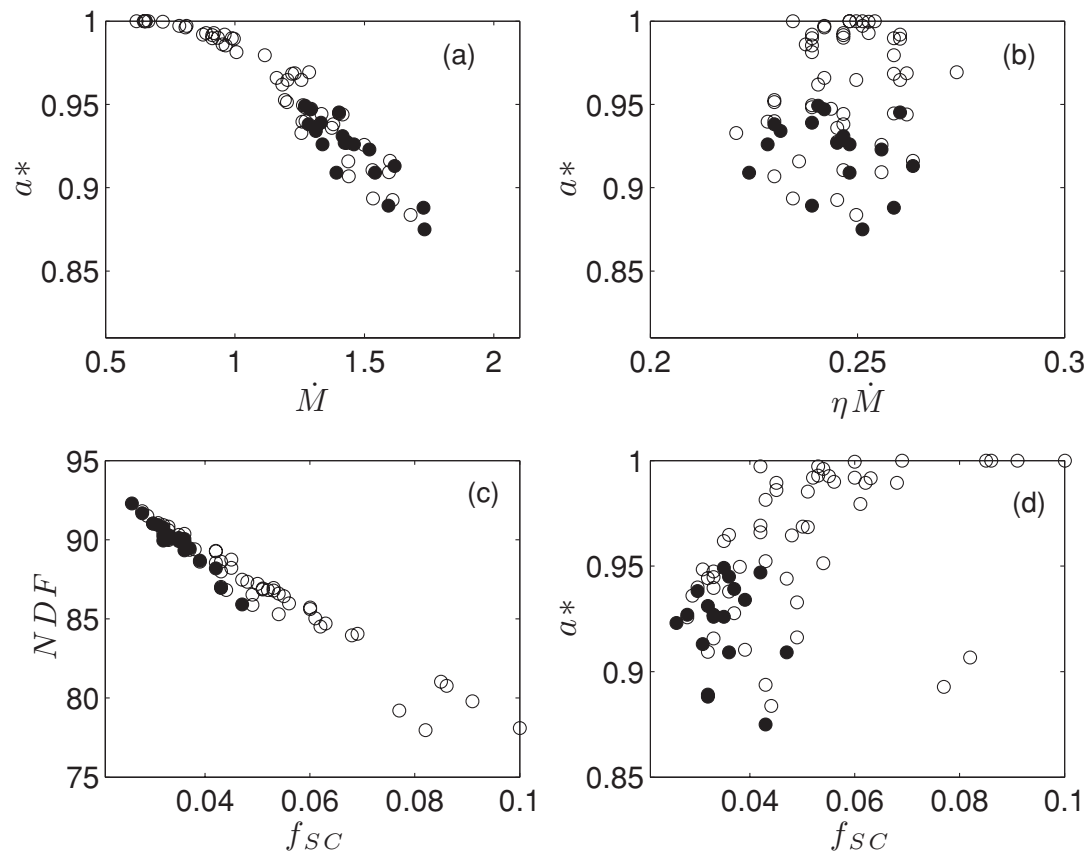

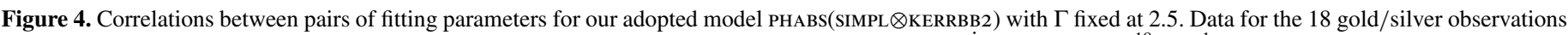

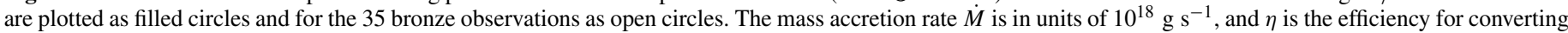
rest mass into radiant energy.

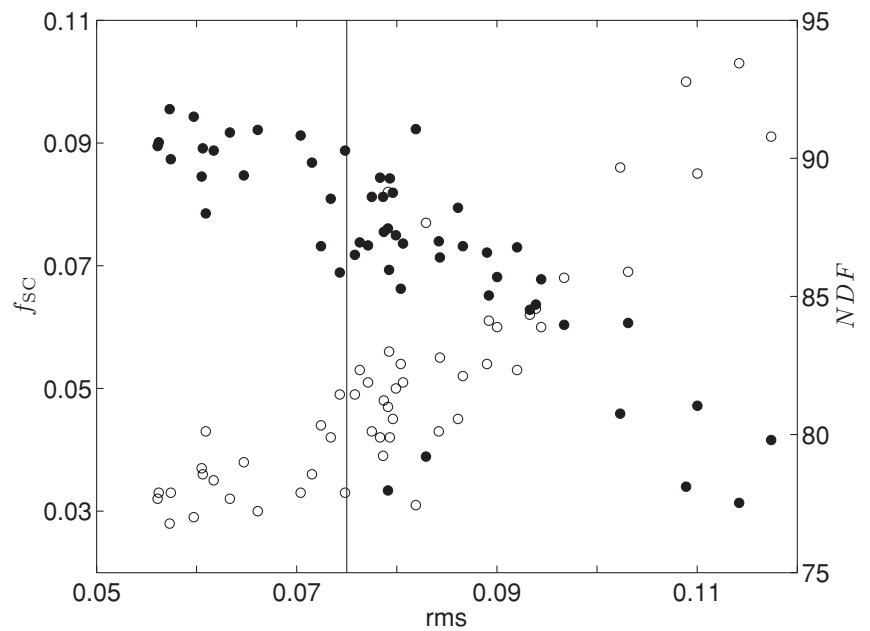

Figure 5. Scattered fraction $f_{\mathrm{SC}}$ (open circles, scale on the left) and the naked disk fraction NDF (filled circles, scale on the right) vs. the rms power (0.1-10 $\mathrm{Hz}$ ) for our standard model PHABS(SIMPL $\otimes$ KERRBB2) with $\Gamma$ fixed at 2.5 . The solid vertical line separates the data for our adopted sample of 18 gold/silver spectra (rms power $<0.075$ ) from that of the 35 bronze spectra. We find very similar correlations for $\Gamma=2.3$ and $\Gamma=2.7$, i.e., the scattered fraction generally increases with increasing $\Gamma$ while the disk fraction decreases. This has the effect of shifting the plots up or down relative to the $\Gamma=2.5$ plot shown here.

could use the naked disk fraction instead of $f_{\mathrm{SC}}$; however, we favor $f_{\text {SC }}$ because it is a readily available fit parameter.) We now investigate this possibility in the case of LMC X-1 using the model PHABS(SIMPL $\otimes$ KERRBB2).

In the top panel of Figure 6, we show the rms power for all 53 spectra plotted in the order of increasing rms power. The horizontal line marks the TD-state criterion rms $<0.075$ and the vertical line separates our 18 gold/silver spectra on the left from the 35 bronze spectra on the right. With the same ordering of the spectra, we show in the lower panel for all 53 spectra the scattered fraction. The dashed line for $f_{\mathrm{SC}}=4 \%$

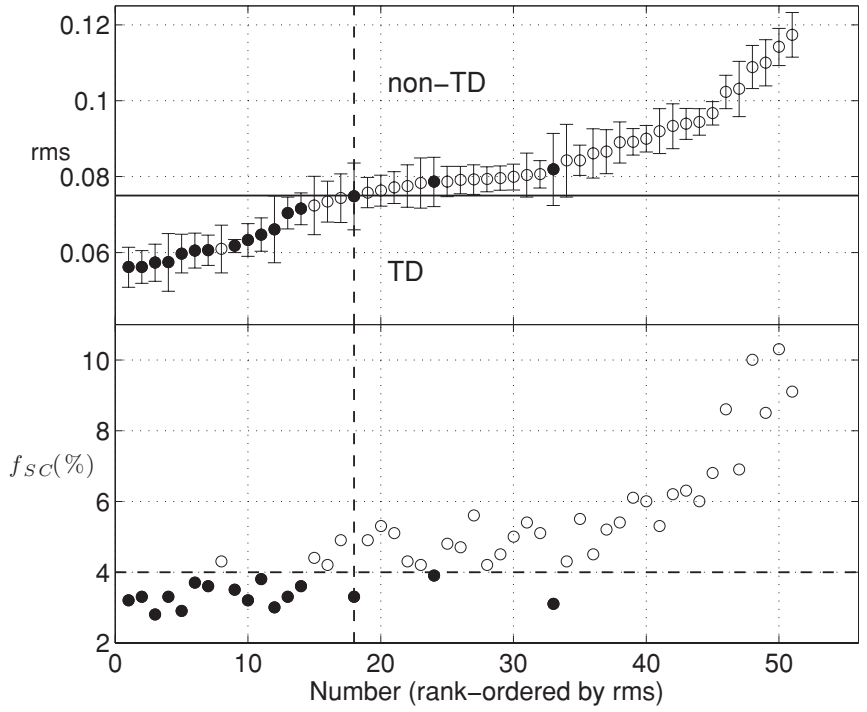

Figure 6. Exploration of the relationship of the classical TD rms criterion ( $\mathrm{rms}$ power $<0.075)$ to the scattered fraction. The spectra are plotted in the order of increasing rms power. Top: the first 18 data points with rms power $<0.075$ (indicated by the vertical dashed line and solid horizontal line) correspond to our gold/silver spectra and the remaining 35 are the bronze spectra. Bottom: the horizontal dashed line indicates the scattered-fraction cut line at $4 \%$ that corresponds to the rms power $=0.075$ line (see the text). The spectra with $f_{\mathrm{SC}}<4 \%$ are indicated by filled circles in the top panel. As shown, there is a very good correspondence between favored spectra with low rms power and those with low scattered fraction.

was determined as follows. We assumed that both the rms power and $f_{\mathrm{SC}}$ follow normal distributions and are uncorrelated with the other fitted parameters. For the rms power we find a mean value of $\mathrm{rms}=0.081 \pm 0.016$ (std. dev.; $N=53$ ), which is $0.38 \sigma$ above the rms $=0.075$ line in the top panel. Given the correlation between $f_{\mathrm{sc}}$ and rms power (Figure 5), we expect the corresponding scattered-fraction cut to also be $0.38 \sigma$ below the mean value, i.e., at $f_{\mathrm{sc}}=4 \%$ (rounded to the nearest 

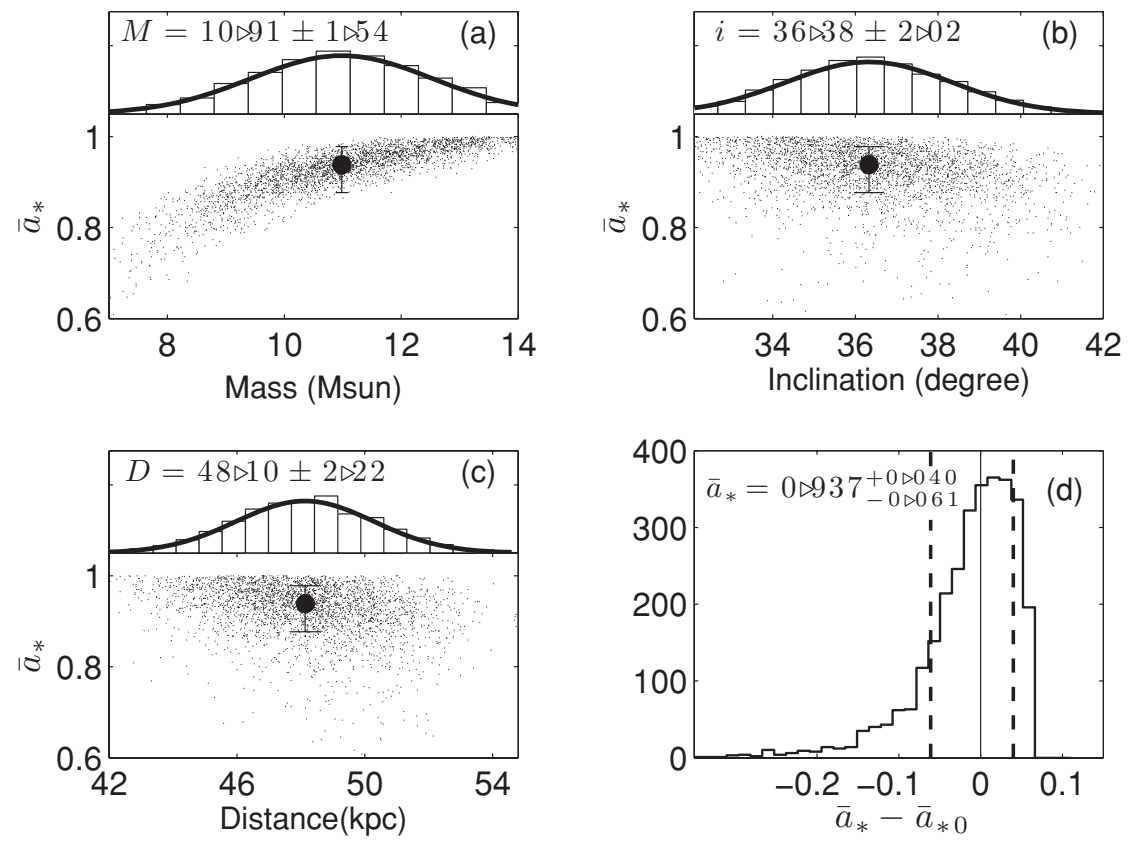

Figure 7. Effect on the spin parameter $a_{*}$ of varying the input parameters $M, i$, and $D$ for the viscosity parameter $\alpha=0.01$. (a) The upper panel shows a normal distribution for the black hole mass and the lower panel shows $a_{*}$ versus mass $M$ for 3000 sets of parameters drawn at random. The central filled circle indicates our estimate of the spin $\left(\bar{a}_{* 0}=0.937_{-0.061}^{+0.040}\right)$ obtained from these simulations. (b, c) Same as panel (a) except now for the parameters of inclination angle and distance, respectively. That the uncertainty in the mass is the dominant source of observational error is indicated in panel (a) by the significant correlation between $a_{*}$ and $M$. (d) Histogram of spin displacements for 3000 parameter sets. The vertical solid line indicates the median value of the spin in the simulated sample, $\bar{a}_{* 0}=0.937$. The two dashed lines enclose $68.27 \%(1 \sigma)$ of the spin values centered on the solid line and imply an observational uncertainty of $(+0.040,-0.061)$.

percent); this is the level indicated by the dashed line in the lower panel.

The solid symbols in the top panel correspond to the 16 spectra in the lower panel that have $f_{\mathrm{sc}}<4 \%$. It can be seen that all of the spectra selected by scattered fraction are consistent or nearly consistent with the TD criterion rms $<0.075$. Likewise, in the lower panel one sees that most (14 out of 18) of the gold/silver spectra have $f_{\text {sc }}<4 \%$ while four of them have $4 \%<f_{\text {sc }} \lesssim 5 \%$. Meanwhile, most of the 35 bronze spectra with rms significantly greater than 0.075 are soundly rejected by the criterion $f_{\mathrm{sc}}<4 \%$.

We now compare the fitting results obtained for the data selected using the criterion rms $<0.075$ to that selected using the criterion $f_{\mathrm{sc}}<4 \%$ and find that they are essentially identical. The spin parameter and the scattered fraction for the rms-selected data are respectively $a_{*}=0.938 \pm 0.020$ and $f_{\mathrm{sc}}=0.036 \pm 0.005$ (Table 2), and for the $f_{\mathrm{SC}}$-selected data are $a_{*}=0.938 \pm 0.016$ and $f_{\mathrm{SC}}=0.033 \pm 0.003$. These results are based only on LMC X-1. However, they indicate that whenever high-quality timing data are unavailable for a source that it may be possible to establish a useful scattered-fraction criterion capable of rejecting data with high flicker noise. Of course, the scattered-fraction cut level that one adopts may vary from source to source.

In Appendix A, we make the first use of our newly defined scattered fraction criterion, $f_{\mathrm{SC}}<4 \%$, in order to select data obtained by five X-ray missions for which the RXTE rms dataselection criterion cannot be applied.

\subsection{Comprehensive Error Analysis: Final Determination of Spin Parameter}

The statistical error in the spin parameter is quite small: $a_{*}=0.938 \pm 0.020(\alpha=0.01$; Table 2$)$. At this level of pre- cision, the observational error is dominated by the uncertainties in the input parameters: the black hole mass $M$, the orbital inclination angle $i$, and the distance $D$ (Section 1; Orosz et al. 2009). In order to determine the error in $a_{*}$ due to the combined uncertainties in $M, i$, and $D$, we performed Monte Carlo simulations assuming that the uncertainties in these parameters are normally and independently distributed. Specifically, we (1) generated 3000 parameter sets for $M, i$, and $D$; (2) computed for each set the look-up table for the spectral hardening factor $f$ using the model BHSPEC; and (3) using these $f$-tables, we obtained $a_{*}$ by fitting our standard model (Section 4.3) to the 18 gold/silver spectra.

The results for the 3000 simulations are shown in Figure 7. Panels (a)-(c) show the distribution of the values of $a_{*}$ for the parameters $M, i$, and $D$, respectively. Each set of 18 spectra is averaged and represented by a single data point. In each panel, the value of the parameter in question is given (Orosz et al. 2009), and a large central dot is plotted among the 3000 distributed points at $a_{*}=0.937$, which is the median value of the distribution. The histogram for the displacements in $a_{*}$ about the most probable value is shown in panel $d$. As indicated, the $1 \sigma$ error in $a_{*}$ is $(+0.040,-0.061)$, which is much larger than the statistical error of \pm 0.020 (Table 2). The error is dominated by the uncertainty in $M$; the uncertainties in $i$ and $D$ are relatively unimportant.

The analysis above is for our standard value of $\alpha=0.01$. We also performed the Monte Carlo analysis for our other fiducial value of the viscosity parameter, $\alpha=0.1$, which as we saw in Section 5.3 results in a slightly lower mean value of the spin parameter, $a_{*}=0.908 \pm 0.023$. (The computed median value of the distribution is $a_{*}=0.907$.) In Figure 8, we show the results of this analysis as a dashed-line histogram of spin displacements; the histogram for $\alpha=0.01$, which is copied from Figure 7(d), is also shown by the smaller solid-line histogram. The summation 


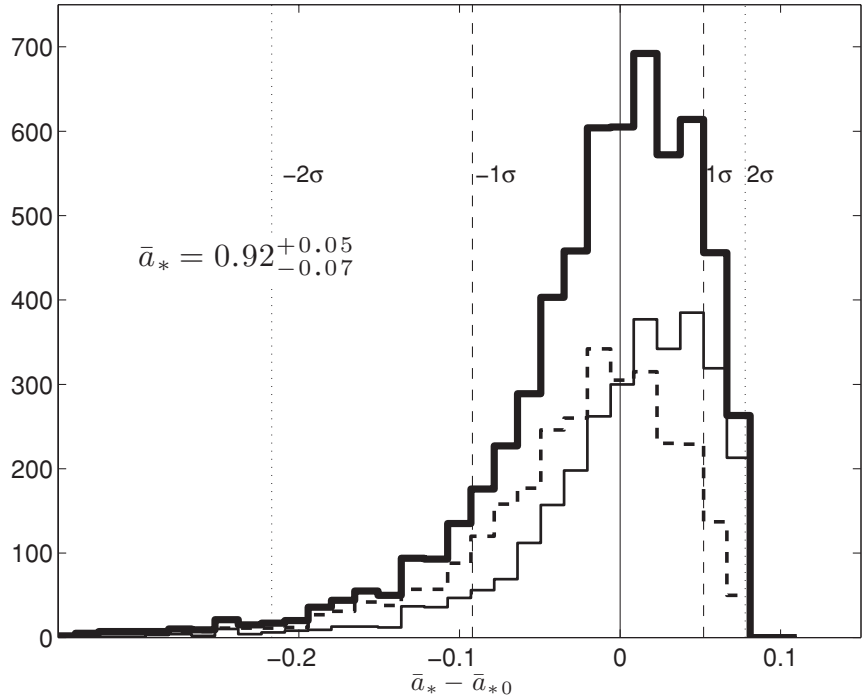

Figure 8. Combined error analysis that considers both of our fiducial values for the viscosity parameter. The thin solid line is for $\alpha=0.01$ and the dashed line is for $\alpha=0.1$. The sum of these two smaller histograms forms the large histogram (thick solid line), which shows the spin displacements for a total of 6000 parameter sets. The vertical solid line indicates the median value of the spin determined by these simulations: $\bar{a}_{* 0}=0.92$. The two dashed lines enclose $68.27 \%(1 \sigma)$ of the spin values centered on the solid line and imply an observational and model-parameter uncertainty of $(+0.05,-0.07)$. The two dotted lines enclose $95.45 \%(2 \sigma)$ of the spin values and imply an uncertainty of $(+0.06,-0.18)$

of these two histograms results in the large histogram (heavy solid line). This combined distribution, which corresponds to a total of 6000 simulations, has a median spin value of 0.92 and implies a $1 \sigma$ error of $(+0.05,-0.07)$. Thus, considering both the uncertainties in $M, i$, and $D$ and the inherent uncertainty in the value of the viscosity parameter we arrive at our final result for the spin of LMC X-1: $a_{*}=0.92_{-0.07}^{+0.05}(1 \sigma)$.

\section{DISCUSSION}

Throughout, we have focused on the three gold spectra, which fully satisfy the TD-state criteria, and 15 additional silver spectra. These latter spectra meet the TD-state rms power criterion. However, they yield low values of the disk fraction (Table 1) that fail to meet the TD-state disk-fraction criterion because the standard power-law model is inadequate for modeling their steep Compton-tail components. We have shown that these 18 gold/silver spectra when analyzed using the Comptonization model SIMPL yield large values of the naked disk fraction (Table 2). Restricting ourselves to this sample of 18 spectra and considering only the statistical error, we obtained our featured value of the spin parameter: $a_{*}=0.938 \pm 0.020$ (std. dev.; $N=18$ ). For comparison, if we consider all 53 spectra (i.e., including the bronze spectra) we obtain: $a_{*}=0.961 \pm 0.033$ (std. dev.; $N=53$; Table 3). That is, we obtain a statistically consistent result, although with a somewhat higher spin and with a larger uncertainty. We have chosen to base our result on just the 18 gold/silver spectra because they meet the TD-state rms criterion and because their spectra are strongly dominated by the thermal component, i.e., their $2-20 \mathrm{keV}$ naked disk fractions are $\gtrsim 86 \%$ when computed using simPL (Table 2). When we include all the sources of observational error and model parameter uncertainties, our final result for the spin parameter is $a_{*}=0.92_{-0.07}^{+0.05}$.

The greatest uncertainty in our spin estimate arises from the uncertainties in the validity of the disk model we employ. For example, the model assumes a razor-thin disk for which the viscous torque is assumed to vanish at the innermost stable circular orbit (ISCO), an assumption that, it has been argued, does not hold in the presence of magnetic fields (Krolik \& Hawley 2002). However, our preliminary hydrodynamic work indicates that the effects of these viscous torques and emission from within the ISCO are modest for the thin disks $(H / R \leqslant 0.1)$

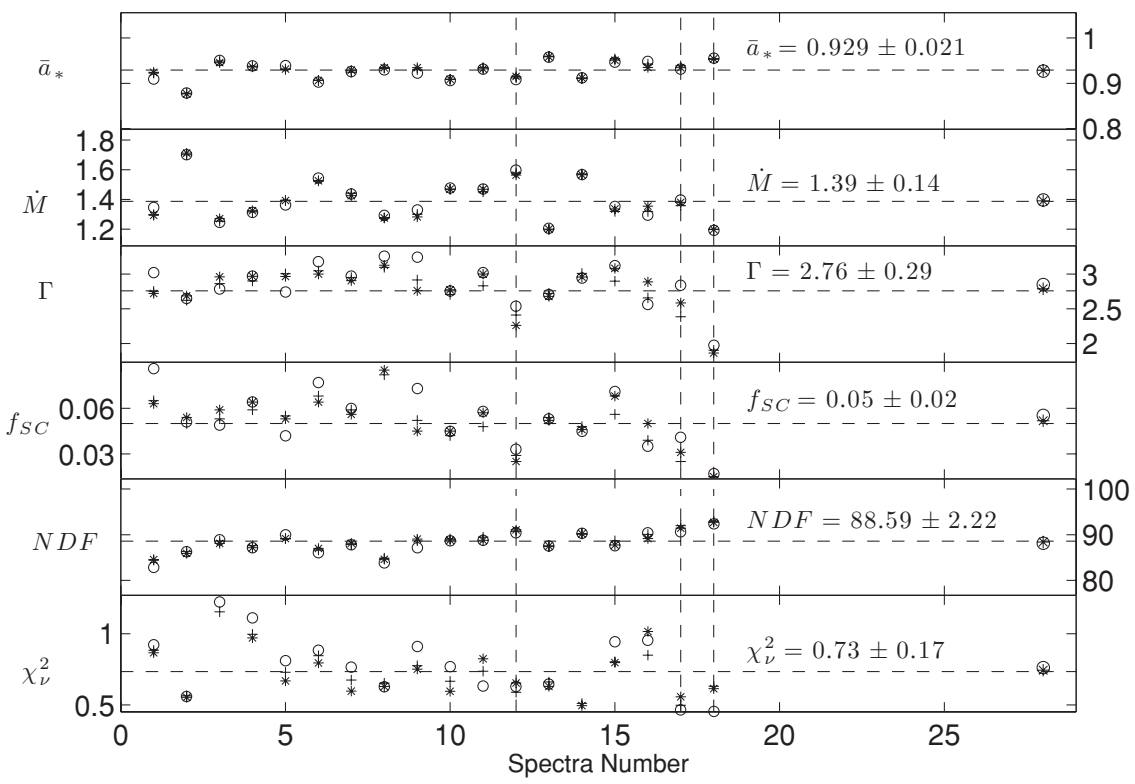

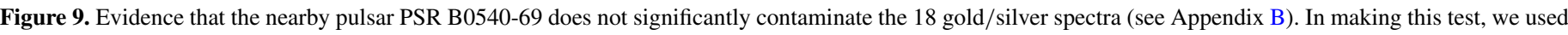

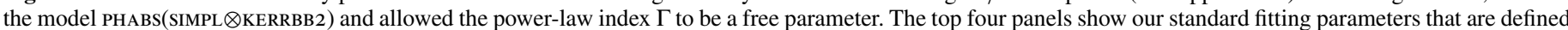

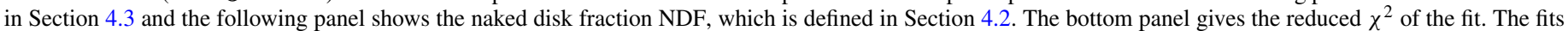

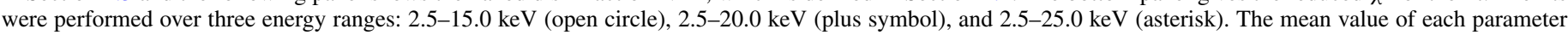

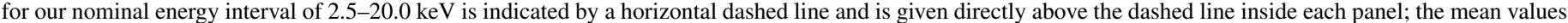

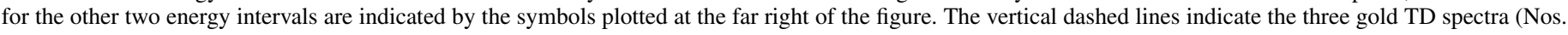
12,17 , and 18) 
and low luminosities $\left(L / L_{\text {Edd }} \leqslant 0.3\right)$ that we restrict ourselves to (McClintock et al. 2006; Shafee et al. 2006). In the case of LMC X-1, with its modest luminosity $\left(L / L_{\text {Edd }} \approx 0.16\right.$; Table 2) and high spin, these hydrodynamic results imply a very small uncertainty, $\Delta a_{*} \lesssim 0.01$ (see Figure 9 in Shafee et al. 2008b). Another uncertainty lies in our assumption that the black hole spin is approximately aligned with the angular momentum vector of the binary. As Figure 3(b) indicates, if any misalignment is $\lesssim 2^{\circ}$, then it will contribute an error in the spin parameter $a_{*}$ that is no larger than our total observational error. A future X-ray polarimetry mission may allow a check on our assumption of alignment (Li et al. 2009).

Our preliminary hydrodynamic results are supported by our more recent three-dimensional general relativistic MHD simulations of an appropriately thin disk with $H / R \sim 0.05-0.1$ (Shafee et al. 2008a). This model is for a nonspinning black hole. In steady state, the specific angular momentum profile of the inflowing magnetized gas deviates by $<2 \%$ from that of the standard thin disk model of Novikov \& Thorne (1973). In addition, the magnetic torque at the ISCO is only $\sim 2 \%$ of the inward flux of angular momentum at this radius. These results indicate that magnetic coupling across the ISCO is relatively unimportant for geometrically thin disks, at least in the case of a nonspinning black hole.

In short, our recent theoretical studies indicate that the disk emission is truncated rather sharply at the ISCO and that measuring the truncation radius provides a reliable estimate of spin. In addition, there is a long history of observational evidence suggesting that fitting the X-ray continuum is a promising approach to measuring black hole spin. This history begins in the mid-1980s with the simple nonrelativistic multicolor disk model (Mitsuda et al. 1984; Makishima et al. 1986) now known as DISKBB, which returns the color temperature $T_{\text {in }}$ at the innerdisk radius $R_{\text {in }}$ (Section 3 ). In their review paper on black hole binaries, Tanaka \& Lewin (1995) summarize examples of the steady decay (by factors of 10-100) of the thermal flux of transient sources during which $R_{\text {in }}$ remains quite constant (see their Figure 3.14). They remark that the constancy of $R_{\text {in }}$ suggests that this fit parameter is related to the radius of the ISCO. Zhang et al. (1997) then outlined how, using a relativistic disk model and corrections for the effects of radiative transfer, the fixed inner disk radius provides an observational basis to infer black hole spin. More recently, this evidence for a constant inner radius in the thermal state has been presented for a number of sources via plots showing that the bolometric luminosity of the thermal component is approximately proportional to $T_{\text {in }}^{4}$ (Kubota et al. 2001; Kubota \& Makishima 2004; Gierliński \& Done 2004; Abe et al. 2005; McClintock et al. 2009).

We now consider the possibility that our results are affected by a component of absorption that is not included in our model. Cooke et al. (2008) have found evidence for an ionization cone within a few parsecs of LMC X-1. They argue that the cone must be powered primarily by photoionization from the accretion disk, but require a 13-300 eV photon flux which exceeds the extrapolated hard X-ray model by a factor $\sim 10$. Both this excess and the anisotropy of the ionized gas suggest that the $\mathrm{X}$-ray emission might be partially obscured near the source, possibly by the outer disk. If this hypothesis is correct, we could be underestimating the true disk luminosity and, therefore, overestimating the spin. However, we stand by our estimate that the bolometric luminosity of the disk at the source is $L / L_{\text {Edd }} \sim 0.16$ (Table 2) while regarding the evidence that the disk is substantially more luminous as tentative for several reasons: e.g., the possibility of accretion rate variations over the recombination time of the ionized gas; the lack of evidence for obscuration in the X-ray spectrum; the close proximity of other external ionizing sources; and the likelihood of significant EUV flux via X-ray reprocessing in the accretion disk. On this last point, our quantitative estimate indicates that reprocessing can provide the ionizing photon flux required to explain the He II $\lambda 4686$ line flux. Using the accretion-disk reprocessing model described in Appendix A of Orosz et al. (2009), and assuming that the annular size of the reprocessor is $R=100-10^{5} \mathrm{GM} / \mathrm{c}^{2}$, we find a $54.4-300 \mathrm{eV}$ photon flux of $\approx 4 \times 10^{46}$ photons s$^{-1}$, which matches the flux that Cooke et al. (2008) find is required to power the ionization cone. ${ }^{8}$

What is the origin of the high spin of LMC X-1's black hole primary? Was the black hole born with its present spin, or was it torqued up gradually via the accretion flow supplied by its companion? In order to achieve a spin of $a_{*} \approx 0.9$ via disk accretion, an initially nonspinning black hole must accrete more than $4 M_{\odot}$ from its donor (King \& Kolb 1999) in becoming the $M=10.9 M_{\odot}$ black hole that we observe today (Orosz et al. 2009). However, to transfer this much mass even in the case of Eddington-limited accretion $\left(\dot{M}_{\mathrm{Edd}} \equiv L_{\mathrm{Edd}} / c^{2} \approx\right.$ $\left.2.50 \times 10^{-8} M_{\odot} \mathrm{yr}^{-1}\right)$ requires $>150$ million years, whereas the age of the system is only about 5 million years (Orosz et al. 2009). Thus, we conclude that the spin of LMC X-1 is natal, which is the same conclusion that has been reached for three other stellar black holes (McClintock et al. 2006; Shafee et al. 2006; Liu et al. 2008). We note, however, that an alternative scenario for the origin of high spins for black holes in X-ray binaries has been proposed that invokes hypercritical accretion (Moreno Méndez et al. 2008).

\section{CONCLUSION}

We find that the spin of the black hole primary in LMC X-1 is $a_{*}=0.92_{-0.07}^{+0.05}$, where the uncertainty includes all sources of observational error and model parameter uncertainties. This result is based on an analysis of three TD spectra, which we refer to as our gold spectra, plus 15 silver spectra. The observations that yielded the 15 silver spectra satisfy the TD criterion that their rms power is $<0.075$. However, when analyzed using the standard power-law model they fail to meet the TD-criterion that their disk fractions are $>75 \%$. We show that the failure of the silver spectra to meet this criterion is caused by the unphysical behavior of the standard power-law model, which diverges at low energies and therefore intrudes on the thermal component of emission. We sidestep this problem by using our Comptonization model sIMPL. If we admit an additional 35 bronze spectra (with rms power $>0.075$ ) into the analysis and consider all 53 RXTE spectra, we obtain a consistent, somewhat higher, and less certain value of the spin parameter. Meanwhile, our spin results for the 18 gold/silver RXTE spectra are confirmed by our analyses of XMM-Newton, BeppoSAX, and Ginga spectra (see Appendix A).

\footnotetext{
8 We obtained the value $4 \times 10^{46}$ by integrating the photon flux, assuming blackbody emission, over the energy range from $54.4 \mathrm{eV}$ to $300 \mathrm{eV}$ and over the entire radial extent of the reprocessing region. We used the radial temperature profile specified by Equations (A3) and (A5) in Appendix A of Orosz et al. (2009). Our result is insensitive to the values we assumed for the inner and outer radii of the annular reprocessor. For example, if we halve the inner radius or double the outer radius, the $54.4 \mathrm{eV}$ to $300 \mathrm{eV}$ photon flux changes by only $\approx 10 \%$.
} 
Table 4

Results for LMC X-1 for Five Other X-ray Missions Using PHABS(SIMPL $\otimes$ KERRBB2) ${ }^{\mathrm{a}}$

\begin{tabular}{|c|c|c|c|c|c|c|c|c|c|c|}
\hline No. & Mission and Detector & $a_{*}$ & $\dot{M}$ & $f$ & $\Gamma$ & $f_{\mathrm{SC}}$ & $\operatorname{NDF}(\%)$ & $\chi_{v}^{2}$ & $L / L_{\mathrm{edd}}$ & $N_{\mathrm{H}}(1.0 \mathrm{E} 22)$ \\
\hline 1 & XMM-Newton EPIC & $0.957 \pm 0.002$ & $0.746 \pm 0.011$ & 1.618 & 2.5 & $0.002 \pm 0.002$ & 99.35 & 0.96 & 0.123 & $0.443 \pm 0.004$ \\
\hline 2 & BeppoSAX MECS & $0.969 \pm 0.006$ & $1.057 \pm 0.039$ & 1.628 & 2.5 & $0.021 \pm 0.003$ & 94.18 & 1.03 & 0.148 & $0.459 \pm 0.031$ \\
\hline 3 & Ginga $\mathrm{LAC}^{\mathrm{a}}$ & $0.914 \pm 0.022$ & $1.487 \pm 0.136$ & 1.606 & 2.5 & $0.023 \pm 0.005$ & 93.20 & 0.97 & 0.159 & $0.453 \pm 0.093$ \\
\hline 4 & BBXRT/Astro-1 & $0.902 \pm 0.028$ & $0.934 \pm 0.067$ & 1.605 & 2.5 & $0.099 \pm 0.019$ & 71.90 & 1.06 & 0.096 & $0.575 \pm 0.015$ \\
\hline 5 & $A S C A$ GIS $^{\mathrm{b}}$ & $0.778 \pm 0.012$ & $2.287 \pm 0.048$ & 1.643 & 2.5 & $0.079 \pm 0.005$ & 78.64 & 0.70 & 0.176 & $0.512 \pm 0.006$ \\
\hline
\end{tabular}

Notes.

${ }^{a}$ In order to fit this Ginga LAC spectrum, it was necessary to add a Gaussian emission-line component, PHABS(SIMPL $\otimes($ KERRBB2 + GAUSSIAN)); the central energy of the line, its FWHM and equivalent width are respectively $7.0 \pm 1.5 \mathrm{keV}, 1.3 \pm 0.6 \mathrm{keV}$, and $0.51 \mathrm{keV}$.

${ }^{\mathrm{b}}$ ASCA SIS gives a similarly low value of $\operatorname{spin} a_{*}=0.742 \pm 0.012$

We thank an anonymous referee for very helpful comments and a thorough reading of our paper. J.E.M. acknowledges support from NASA grant NNX08AJ55G. J.F.L. acknowledges support provided by NASA through grant HST-GO-11227 and the Chandra Fellowship Program, grant PF6-70043. R.N. acknowledges support from NASA grant NNX08AH32G and NSF grant AST-0805832. J.F.S. was supported by the Smithsonian Endowment Funds. S.W.D. acknowledges support through the Chandra Fellowship Program, grant PF6-70043. R.A.R. acknowledges support from the NASA contract to MIT for the instrument team contributing to $R X T E$. This research has made use of data obtained from the High Energy Astrophysics Science Archive Research Center (HEASARC) at NASA/Goddard Space Flight Center.

\section{APPENDIX A}

\section{DETERMINATIONS OF THE SPIN PARAMETER FOR FIVE ADDITIONAL X-RAY MISSIONS}

As stated in the final paragraph of Section 5.1, our featured result for the 18 gold/silver $R X T E$ spectra for the case $\alpha=0.01$, $a_{*}=0.938 \pm 0.020$ (std. dev.; $N=18$ ), is confirmed by an analysis of data from three other X-ray missions. In the paragraphs below, which are prefaced by a mission name, we consider in turn X-ray spectral data for LMC X-1 that were obtained during the course of the five missions named in Table 4 (Column 2). With the exception of the BBXRT and Ginga data, all the data were downloaded from the NASA HEASARC. As discussed below, after applying the data selection criterion developed in Section 5.5, namely scattered fraction $f_{\mathrm{SC}}<4 \%$, we find that only three of these missions provide reliable estimates of spin (Table 4, lines 1-3), i.e., estimates of spin that are comparable in quality to the 18 gold/silver $R X T E$ PCA spectra. As in the analysis of the RXTE data, for all five of these missions we fix the power-law index at 2.5 , which is a necessity for four of the five missions whose detectors are unresponsive above $\sim 10 \mathrm{keV}$. As indicated below, small corrections were applied to the effective areas of the various detectors to align them with the standard Toor \& Seward (1974) spectrum of the Crab. We consider here only the case $\alpha=0.01$.

XMM-Newton. A single $4940 \mathrm{~s}$ observation was performed on UT 2000 October 21 using the EPIC-pn camera in timing mode. The data, which are free of pile-up effects, were reduced using precisely the procedures recommended for timing data. ${ }^{9}$ We fitted the data over the energy range 1.0-10.0 keV (Wilms et al. 2003) and corrected the detector's effective area by the

\footnotetext{
9 ftp://ftp.xray.mpe.mpg.de/xmm/service/data_analysis/EPIC_PN/timing/ timing_mode.html
}

factor 0.889 (i.e., the effective area is increased by dividing by the factor 0.889), which is based on observations of the Crab (Kirsch et al. 2005). Conclusion: the data meet the requirement $f_{\mathrm{SC}}<4 \%$, and the spin is consistent with the $R X T E$ value to within 0.9 standard deviations (Table 4 , line 1).

BeppoSAX. A single observation was made on UT 1997 October 5 using the narrow-field instruments aboard BeppoSAX. The four detector systems, as well as this specific observation of LMC X-1, are described in detail in Haardt et al. (2001). We have chosen to analyze only the data obtained by the Medium Energy Concentrator Spectrometer (MECS = MECS2 + MECS3) because the PDS and HPGSPC spectra are noisy and the LECS spectrum lacks a proper response file. The MECS data have been reduced following the standard procedures described in the Cookbook for the BeppoSAX NFI Spectral Analysis. ${ }^{10}$ The net exposure time was $40 \mathrm{ks}$. The spectra obtained by the two MECS detectors, MECS2 and MECS3, were fitted jointly over the energy range $1.65-10.5 \mathrm{keV}$; no correction to the effective area was required. Conclusion: the data meet the requirement $f_{\mathrm{SC}}<4 \%$, and the spin is consistent with the $R X T E$ value to within 1.5 standard deviations (Table 4, line 2).

Ginga. The Ginga Large Area Proportional Counter (LAC; 1.2-37 keV) observed LMC X-1 on six UT dates: 1987 April 22, 1987 July 16, 1987 September 30, 1988 August 15, 1990 June 21, and 1991 February 15. The respective exposure times were approximately $7290,12831,4841,315,326$, and $266 \mathrm{~s}$. We extracted the spectra following the procedures described in Ebisawa et al. (1989). We applied to each spectrum an effectivearea correction factor of 0.968 based on an observation of the Crab (Turner et al. 1989). We fitted all six spectra; however, in Table 4 we report our results only for the spectrum obtained on 1987 September 30, the only spectrum that satisfies our data selection criterion $\left(f_{\mathrm{SC}}<4 \%\right)$. This spectrum was fitted over the restricted energy range $1.2-12.0 \mathrm{keV}$ because the spectrum contains large and unexplained residual features at higher energies. Conclusion. The data meet the requirement $f_{\mathrm{SC}}<4 \%$, and the spin is consistent with the $R X T E$ value to within 0.8 standard deviations (Table 4 , line 3 ).

BBXRT/Astro-1. Four observations were performed on UT 1990 December 6 and 7. We disregard the observations on December 7 because of their high background levels and large off-axis angles; we consider only the pair of observations performed on December 6 with exposure times of $1235 \mathrm{~s}$ and 616 $\mathrm{s}$. The data have been reduced following the standard procedures (Schlegel et al. 1994). The two spectra were fitted jointly over the energy range $0.7-7.0 \mathrm{keV}$, and the effective area was corrected by the factor 0.973 (Weaver et al. 1995). Conclusion: The

\footnotetext{
10 http://heasarc.gsfc.nasa.gov/docs/sax/abc/saxabc/saxabc.html
} 
scattered fraction fails to meet the requirement $f_{\mathrm{SC}}<4 \%$, the spin value is unreliable, and we disregard it (Table 4, line 4). However, we note that the BBXRT spin value is consistent with the RXTE value to within 1.0 standard deviation.

ASCA. A single 3465 s observation was performed on UT 1995 April 2. We consider only the data obtained by the Gas Imaging Spectrometer (GIS). We extracted the spectra following the standard procedures described in the ASCA ABC guide. ${ }^{11}$ The two GIS spectra (GIS2 and GIS3) were fitted jointly over the energy range $0.7-8.0 \mathrm{keV}$. No correction to the effective area is required because the GIS effective area calibrations were based on the Toor \& Seward (1974) spectrum of the Crab. Conclusion: the scattered fraction fails to meet the requirement $f_{\mathrm{SC}}<4 \%$, and the spin value is unreliable. We therefore disregard it while noting that it is exceptionally low, a result for which we have no explanation.

\section{APPENDIX B}

\section{CONTAMINATION BY THE PULSAR PSR B0540-69}

The pulsar PSR B0540-69 is located 25' from LMC X-1. Haardt et al. (2001) concluded that their BeppoSAX PDS spectra (15-100 keV) of LMC X-1 were contaminated by the presence of this nearby pulsar. Even though the PCA has a narrower field of view than the BeppoSAX PDS, 1.0 versus 1.5 (FWHM), our spectra of LMC X-1 could likewise be significantly contaminated. We now describe three lines of evidence showing that this is not the case. We first estimate the contribution of the pulsar to the total flux using its welldetermined spectrum; we then search for the expected signature of the pulsar's hard spectrum in our data; and lastly we subtract a faked version of the pulsar's spectrum from the source spectrum. (We disregard PSR J0537-6910, which also lies in the PCA field of view, because it is further off-axis and significantly fainter than PSR B0540-69; Cusumano et al. 1998).

PSR B0540-69 and its 2" -3 " plerion nebula were discovered 25 years ago by the Einstein X-ray observatory (Seward et al. 1984). The source has since been closely observed by many missions (e.g., ROSAT, RXTE, Chandra, and INTEGRAL). It is quite similar to the Crab. Like the Crab, all studies to date show that its hard spectrum can be fitted with a single power law with a photon index $\Gamma$ in the range 1.8-2.2 (Sunyaev et al. 1990; Kaaret et al. 2001; Götz et al. 2006). On the other hand, the power-law component in the spectrum of LMC X-1, is much softer, $\Gamma \sim 2.5-3$ (Table 1; Nowak et al. 2001; Haardt et al. 2001). We now estimate at what energy the flux in the flat pulsar spectrum first exceeds the flux of LMC X-1. With LMC X-1 centered in the PCA, the collimator transmission factor for the pulsar is $T=0.57$. Adopting for the pulsar $\Gamma=2$ and a flux at 1 $\mathrm{keV}$ of $A=8.86 \times 10^{-3} \mathrm{ph} \mathrm{cm}^{-2} \mathrm{~s}^{-1} \mathrm{keV}^{-1}$ (based on the flux observed by INTEGRAL in 2003; Götz et al. 2006), the expected pulsar photon flux is $8.9 \times 10^{-3} \times T \times E^{-2}=5.05 \times 10^{-3} E^{-2}$. For LMC X-1 we adopt $\Gamma=2.5$ and $A=0.2$ (a typical value in Table 1), for which the corresponding photon flux is $0.2 \times E^{-2.5}$. Equating the two fluxes, the flux of the pulsar dominates over that of LMC X-1 only at energies $\gtrsim 1 \mathrm{MeV}$; even for $\Gamma$ (LMC $\mathrm{X}-1)=3$, the pulsar dominates only for energies $\gtrsim 40 \mathrm{keV}$.

We now describe a second test performed on our 18 gold/ silver spectra which further demonstrates that the flux from the nearby pulsar is unimportant. In addition to fitting the spectra over our adopted energy range of $2.5-20.0 \mathrm{keV}$ (Sections 3

\footnotetext{
11 http://heasarc.gsfc.nasa.gov/docs/asca/abc/abc.html
}

and 4), we also fitted them over the ranges $2.5-15.0 \mathrm{keV}$ and $2.5-25.0 \mathrm{keV}$ using precisely the same model and procedures. The results are displayed in Figure 9 where it can be seen that the fit parameters and the values of $\chi_{v}^{2}$ are scarcely affected by the choice of the energy range. This is not at all what one would expect if the spectrum of LMC X-1 was contaminated by a significant component of flux from the pulsar. Rather, one would expect the fit results to vary as the upper energy bound is increased from 15 to $25 \mathrm{keV}$ because the pulsar's spectrum is significantly harder than that of LMC X-1 (see above). Specifically, one would expect the influence of the pulsar to be most apparent at $25 \mathrm{keV}$. However, the effect of the pulsar on our results, if any, are seen to be negligible. In particular, the values of $a_{*}$ (top panel) are completely insensitive to the choice of the fitting range.

As a final direct test, we created a "faked" spectrum of the pulsar in XSPEC using the observed flux and power-law index given above and including the off-axis transmission factor $(T=$ 0.57 ). We then subtracted this simulated spectrum from each of the 18 gold/silver spectra, redid our standard analysis and found $a_{*}=0.947 \pm 0.020$, which differs by only 0.3 standard deviations from our adopted result $\left(a_{*}=0.938 \pm 0.020\right.$; Section 5.1 ), which was obtained by ignoring the flux of the pulsar.

We thus conclude, based on the three arguments given above, that the contaminating effect of the pulsar on our estimate of the spin parameter of LMC X-1 is negligible $(\lesssim 1 \%)$.

\section{REFERENCES}

Abe, Y., Fukazawa, Y., Kubota, A., Kasama, D., \& Makishima, K. 2005, PASJ, 57,629

Arnaud, K. A. 1996, in ASP Conf. Ser. 101, Astronomical Data Analysis Software and Systems V, ed. G. H. Jacoby \& J. Barnes (San Francisco, CA: ASP), 17

Cooke, R., Bland-Hawthorn, J., Sharp, R., \& Kuncic, Z. 2008, ApJ, 687, L29

Cui, W., Feng, Y. X., Zhang, S. N., Bautz, M. W., Garmire, G. P., \& Schulz, N. S. 2002, ApJ, 576, 357

Cusumano, G., Israel, G. L., Mannucci, F., Masetti, N., Mineo, T., \& Nicastro, L. 1998, A\&A, 337, 772

Dal Fiume, D., Haardt, F., Galli, M. R., Treves, A., \& Chiappetti, L. 2001, in AIP Conf. Proc. 599, X-Ray Astronomy: Stellar Endpoints, AGN, and the Diffuse X-Ray Background, ed. N. E. White, G. Malaguti, \& G. G. C. Palumbo (Melville, NY: AIP), 594

Davis, S. W., Blaes, O. M., Hubeny, I., \& Turner, N. J. 2005, ApJ, 621, 372

Davis, S. W., \& Hubeny, I. 2006, ApJS, 164, 530

Done, C., \& Davis, S. W. 2008, ApJ, 683, 389

Dopita, M. A., \& Sutherland, R. S. 2003, Astrophysics of the Diffuse Universe (Berlin: Springer)

Ebisawa, K., Mitsuda, K., \& Inoue, H. 1989, PASJ, 41, 519

Fromang, S., \& Papaloizou, J. 2007, A\&A, 476, 1113

Gierliński, M., \& Done, C. 2004, MNRAS, 347, 885

Götz, D., Mereghetti, S., Merlini, D., Sidoli, L., \& Belloni, T. 2006, A\&A, 448, 873

Haardt, F., et al. 2001, ApJS, 133, 187

Hawley, J. F., \& Krolik, J. H. 2001, ApJ, 548, 348

Hutchings, J. B., Crampton, D., \& Cowley, A. P. 1983, ApJ, 275, L43

Hutchings, J. B., Crampton, D., Cowley, A. P., Bianchi, L., \& Thompson, I. B. 1987, AJ, 94, 340

Jahoda, K., Markwardt, C. B., Radeva, Y., Rots, A. H., Stark, M. J., Swank, J. H., Strohmayer, T. E., \& Zhang, W. 2006, ApJS, 163, 401

Kaaret, P., et al. 2001, ApJ, 546, 1159

King, A. R., \& Kolb, U. 1999, MNRAS, 305, 654

King, A. R., Pringle, J. E., \& Livio, M. 2007, MNRAS, 376, 1740

Kirsch, M. G., et al. 2005, Proc. SPIE, 5898, 22

Krolik, J. H., \& Hawley, J. F. 2002, ApJ, 573, 754

Kubota, A., \& Makishima, K. 2004, ApJ, 601, 428

Kubota, A., Makishima, K., \& Ebisawa, K. 2001, ApJ, 560, L147

Lasota, J.-P. 2001, New Astron. Rev., 45, 449

Leong, C., Kellogg, E., Gursky, H., Tananbaum, H., \& Giacconi, R. 1971, ApJ, 170, L67 
Li, L. X., Narayan, R., \& McClintock, J. E. 2009, ApJ, 691, 847

Li, L. X., Zimmerman, E. R., Narayan, R., \& McClintock, J. E. 2005, ApJS, 157,335

Liu, J., McClintock, J. E., Narayan, R., Davis, S. W., \& Orosz, J. A. 2008, ApJ, 679, L37

Makishima, K., Maejima, Y., Mitsuda, K., Bradt, H. V., Remillard, R. A., Tuohy, I. R., Hoshi, R., \& Nakagawa, M. 1986, ApJ, 308, 635

Mark, H., Price, R., Rodrigues, R., Seward, F. D., \& Swift, C. D. 1969, ApJ, $155, \mathrm{~L} 143$

McClintock, J. E., Narayan, R., \& Shafee, R. 2009, in Black Holes, ed. M. Livio \& A. Koekemoer (Cambridge: Cambridge Univ. Press), in press (arXiv:0707.4492v1)

McClintock, J. E., \& Remillard, R. A. 2006, in Compact Stellar X-Ray Sources, ed. W. H. G. Lewin \& M. van der Klis (Cambridge: Cambridge Univ. Press), 157

McClintock, J. E., Shafee, R., Narayan, R., Remillard, R. A., Davis, S. W., \& Li, L. X. 2006, ApJ, 652, 518

Miller, J. M., Reynolds, C. S., Fabian, A. C., Miniutti, G., \& Gallo, L. C. 2009, ApJ, 697, 900

Miller, J. M., et al. 2002, ApJ, 570, L69

Miller, J. M., et al. 2004, ApJ, 606, L131

Mitsuda, K., et al. 1984, PASJ, 36, 741

Moreno Méndez, E., Brown, G. E., Lee, C. H., \& Park, I. H. 2008, ApJ, 689 , L9

Novikov, I. D., \& Thorne, K. S. 1973, in Black Holes (Les Astres Occlus), ed. C. DeWitt \& B. S. DeWitt (New York: Gordon and Breach), 343

Nowak, M. A., Wilms, J., Heindl, W. A., Pottschmidt, K., Dove, J. B., \& Begelman, M. C. 2001, MNRAS, 320, 316

Orosz, J. A., et al. 2007, Nature, 449, 872

Orosz, J. A., et al. 2009, ApJ, 697, 573
Pessah, M. E., Chan, C. K., \& Psaltis, D. 2007, ApJ, 668, L51

Price, R. E., Groves, D. J., Rodrigues, R. M., Seward, F. D., Swift, C. D., \& Toor, A. 1971, ApJ, 168, L7

Remillard, R. A., \& McClintock, J. E. 2006, ARA\&A, 44, 49

Schlegel, E. M., Marshall, F. E., Mushotzky, R. F., Smale, A. P., Weaver, K. A., Serlemitsos, P. J., Petre, R., \& Jahoda, K. M. 1994, ApJ, 422, 243

Seward, F. D., Harnden, Jr, F. R., \& Helfand, D. J. 1984, ApJ, 287, L19

Shafee, R., McClintock, J. E., Narayan, R., Davis, S. W., Li, L. X., \& Remillard, R. A. 2006, ApJ, 636, L113

Shafee, R., McKinney, J. C., Narayan, R., Tchekhovskoy, A., Gammie, C. F., \& McClintock, J. E. 2008a, ApJ, 687, L25

Shafee, R., Narayan, R., \& McClintock, J. E. 2008b, ApJ, 676, 549

Shapiro, S. L., \& Teukolsky, S. A. 1983, Black Holes, White Dwarfs and Neutron Stars: The Physics of Compact Objects (New York: Wiley)

Steiner, J. F., Narayan, R., McClintock, J. E., \& Ebisawa, K. 2009, PASP, submitted (arXiv:0810.1758v2)

Sunyaev, R. A., \& Titarchuk, L. G. 1980, A\&A, 86, 121

Sunyaev, R., et al. 1990, Sov. Astron. Lett., 16, 55

Swank, J. H. 1999, Nucl. Phys. B Proc. Suppl., 69, 12

Tanaka, Y., \& Lewin, W. H. G. 1995, in X-Ray Binaries, ed. W. H. G. Lewin, J. van Paradijs, \& E. P. J. van den Heuvel (Cambridge: Cambridge Univ. Press), 126

Toor, A., \& Seward, F. D. 1974, AJ, 79, 995

Turner, M. J. L., et al. 1989, PASJ, 41, 345

Weaver, K. A., et al. 1995, ApJS, 96, 303

Wilms, J., Nowak, M. A., Pottschmidt, K., Staubert, R., Kendziorra, E., Gleissner, T., \& Predehl, P. 2003, in New Views on Microquasars, ed. P. Durouchoux, Y. Fuchs, \& J. Rodriguez (Kolkata: Center for Space Physics), 49

Zhang, S. N., Cui, W., \& Chen, W. 1997, ApJ, 482, L155 